\title{
Multiple bumps can enhance robustness to noise in continuous attractor networks
}

\author{
Raymond Wang ${ }^{1,2}$ and Louis Kang*2 \\ ${ }^{1}$ Redwood Center for Theoretical Neuroscience, University of California, Berkeley \\ ${ }^{2}$ Neural Circuits and Computations Unit, RIKEN Center for Brain Science
}

22 February 2022

\begin{abstract}
A central function of continuous attractor networks is encoding coordinates and accurately updating their values through path integration. To do so, these networks produce localized bumps of activity that move coherently in response to velocity inputs. In the brain, continuous attractors are believed to underlie grid cells and head direction cells, which maintain periodic representations of position and orientation, respectively. These representations can be achieved with any number of activity bumps, and the consequences of having more or fewer bumps are unclear. We address this knowledge gap by constructing 1D ring attractor networks with different bump numbers and characterizing their responses to three types of noise: fluctuating inputs, spiking noise, and deviations in connectivity away from ideal attractor configurations. Across all three types, networks with more bumps experience less noise-driven deviations in bump motion. This translates to more robust encodings of linear coordinates, like position, assuming that each neuron represents a fixed length no matter the bump number. Alternatively, we consider encoding a circular coordinate, like orientation, such that the network distance between adjacent bumps always maps onto 360 degrees. Under this mapping, bump number does not significantly affect the amount of error in the coordinate readout. Our simulation results are intuitively explained and quantitatively matched by a unified theory for path integration and noise in multi-bump networks. Thus, to suppress the effects of biologically relevant noise, continuous attractor networks should employ more bumps when encoding linear coordinates; this advantage disappears when encoding circular coordinates. Our findings provide motivation for the presence of multiple bumps in the mammalian grid network.
\end{abstract}

\section{Introduction}

Continuous attractor networks (CANs) sustain a set of activity patterns that can be smoothly morphed from one to another along a low-dimensional manifold (Amari, 1977; Ermentrout and Cowan, 1979; Milnor, 1985). Network activity is typically localized into attractor bumps, whose positions along the manifold can represent the value of a continuous variable. These positions can be set by external stimuli, and their persistence serves as a memory of the stimulus value. Certain CAN architectures are also capable of a feature called path integration. Instead of receiving the stimulus value directly, the network receives its changes and integrates over them by synchronously moving the attractor bump (Cannon et al., 1983; McNaughton et al., 1991; Seung, 1996). Path integration allows systems to estimate an external state based on internally perceived changes, which is useful in the absence of ground truth.

\footnotetext{
*louis.kang@riken.jp
} 
A

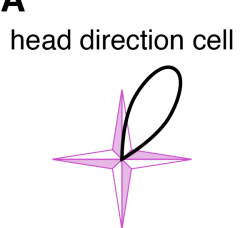

can be produced by coupling orientation with any number of bumps

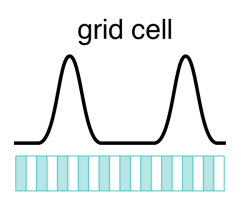

can be produced by coupling position with any number of bumps

\section{B}
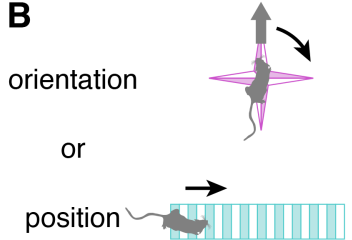

C
one-bump
network

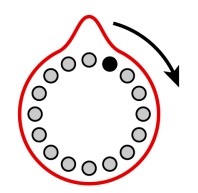

or

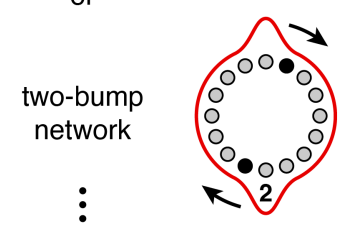

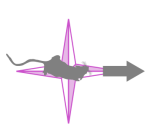
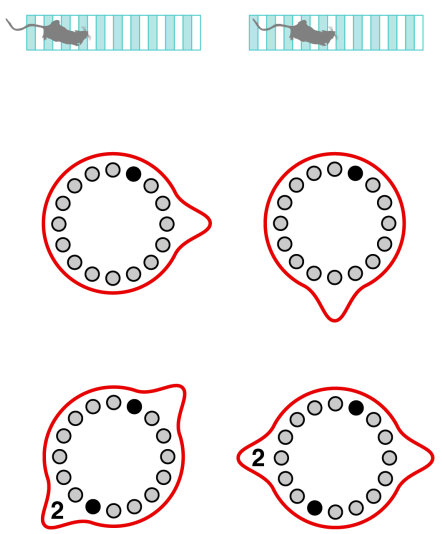
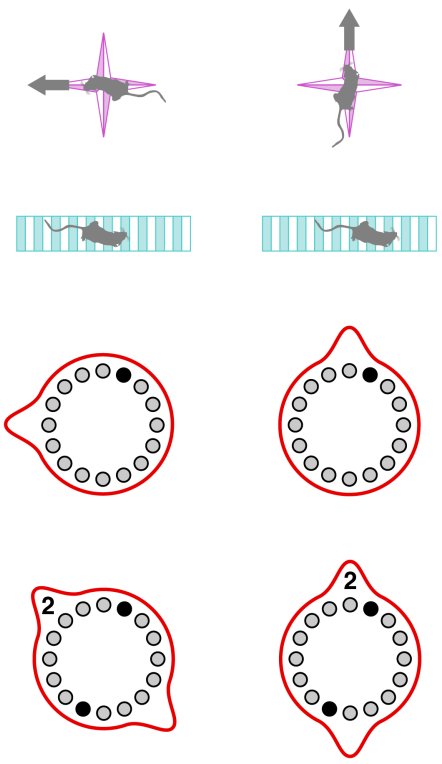

Figure 1: Continuous attractor networks with any number of bumps can produce head direction cells and grid cells. (A) Desired tuning curves of a head direction cell and a 1D grid cell. (B) Orientation and position coordinates whose changes drive bump motion. (C) One- and two-bump ring attractor networks. Each black neuron produces the desired tuning curves in $\mathbf{A}$. In the two-bump network, the coupling to coordinate changes is half as strong, and the second bump is labeled for clarity.

The brain uses path-integrating CANs to encode physical coordinates. Head direction cells in mammals and compass neurons in insects encode spatial orientation by preferentially firing when the animal faces a particular direction relative to landmarks (Fig. 1A, top; Taube et al. 1990; Seelig and Jayaraman 2015). They achieve this as members of 1D CANs whose attractor manifolds have ring topologies (Skaggs et al., 1995; Zhang, 1996). For the case of compass neurons, a ring structure also exists anatomically, and its demonstration of continuous attractor dynamics is well-established (Seelig and Jayaraman, 2015; Kim et al., 2017; Turner-Evans et al., 2017; Green et al., 2017). Grid cells in mammals encode position by preferentially firing at locations that form a triangular lattice in 2D space (1D analogue in Fig. 1A, bottom; Hafting et al. 2005). They are thought to form a 2D CAN with toroidal topology (McNaughton et al., 2006; Fuhs and Touretzky, 2006; Guanella et al., 2007; Burak and Fiete, 2009), and mounting experimental evidence supports this theory (Yoon et al., 2013; Gu et al., 2018; Gardner et al., 2019, 2022). The ability for head direction cells, compass neurons, and grid cells to maintain their tunings in darkness without external cues demonstrates that these CANs can path integrate (Goodridge et al., 1998; Seelig and Jayaraman, 2015; Hafting et al., 2005).

CANs also appear in studies of other brain regions and neural populations. Signatures of continuous attractor dynamics have been detected in the prefrontal cortex during spatial working memory tasks (Constantinidis and Wang, 2004; Wimmer et al., 2014). Theorists have further invoked CANs to explain place cells (Tsodyks and Sejnowski, 1995; Samsonovich and McNaughton, 1997), hippocampal view cells (Stringer et al., 2005), eye tracking (Cannon et al., 1983; Seung, 1996), visual orientation tuning (Ben-Yishai et al., 1995; Somers et al., 1995), and perceptual decision making (Brody et al., 2003; Machens et al., 2005). Thus, CANs are a crucial circuit motif throughout the brain, and better understanding their performance would 
provide meaningful insights into neural computation.

One factor that strongly affects the performance of CANs in path integration is biological noise. To accurately represent physical coordinates, attractor bumps must move in precise synchrony with the animal's trajectory. Hence, the bump velocity must remain proportional to the driving input that represents coordinate changes (Burak and Fiete, 2009). Different sources of noise produce different types of deviations from this exact relationship, all of which lead to path integration errors. While noisy CANs have been previously studied (Tsodyks and Sejnowski, 1995; Zhang, 1996; Compte et al., 2000; Stringer et al., 2002; Renart et al., 2003; Wu et al., 2008; Burak and Fiete, 2009; Itskov et al., 2011; Burak and Fiete, 2012; Kilpatrick and Ermentrout, 2013; Seeholzer et al., 2019), these works did not investigate of role of bump number. CANs with different connectivities can produce different numbers of attractor bumps, which are equally spaced throughout the network and perform path integration by moving in unison (Stringer et al., 2004; Fuhs and Touretzky, 2006; Burak and Fiete, 2009). Two networks with different bump numbers have the same representational capability (Fig. 1). They can share the same attractor manifold and produce neurons with identical tuning curves, as long as the coupling strength between bump motion and driving input scales appropriately. The computational advantages of having more or fewer bumps are unknown.

Our aim is to elucidate the relationship between bump number and robustness to noise. We first develop a rigorous theoretical framework for studying 1D CANs that path integrate and contain multiple bumps. Our theory predicts the number, shape, and speed of bumps. We then introduce three forms of noise. The first is Gaussian noise added to the total synaptic input, which can represent fluctuations in a broad range of cellular processes occurring at short timescales. The second is Poisson spiking noise. The third is noise in synaptic connectivity strengths. The ability for bumps to respond readily to driving inputs is generally conferred by a precise network architecture. We add Gaussian noise to the ideal connectivity and evaluate path integration in this setting. The first two forms of noise are independent over time and neurons, in contrast to the third. We find that networks with more bumps can better resist all three forms of noise under certain encoding assumptions. These observations are explained by our theoretical framework with simple scaling arguments. The following Results section presents all simulation findings and major theoretical conclusions; complete theoretical derivations are found in the Theoretical model section.

\section{Results}

\section{Bump formation in a ring attractor network}

We study a 1D ring attractor network that extends the model of Xie et al. (2002) to allow for multiple attractor bumps. It contains two neural populations $\alpha \in\{\mathrm{L}, \mathrm{R}\}$ at each network position $x$, with $N$ total neurons in each population (Fig. 2A). Each neuron is described by its total synaptic input $g$ that obeys the following dynamics:

$$
\tau \frac{\mathrm{d} g_{\alpha}(x, t)}{\mathrm{d} t}+g_{\alpha}(x, t)=\sum_{\beta} \int \mathrm{d} y W_{\beta}(x, y) s_{\beta}(y, t)+A \pm_{\alpha} \gamma b(t)+\zeta_{\alpha}(x, t)
$$




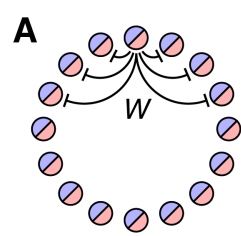

$O$ L neurons $\Delta R$ neurons

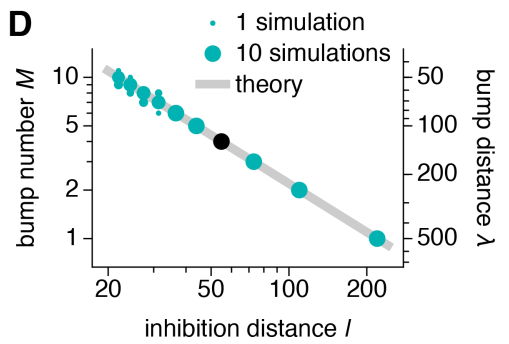

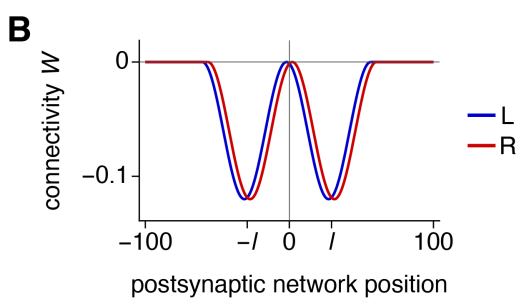

E

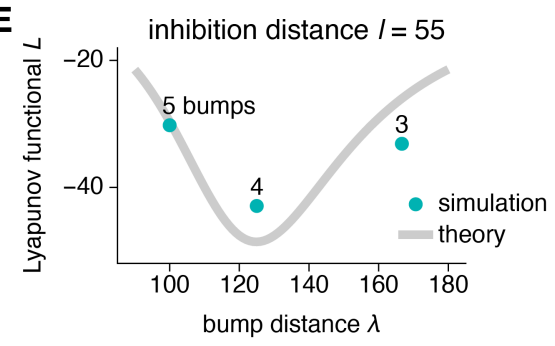

C
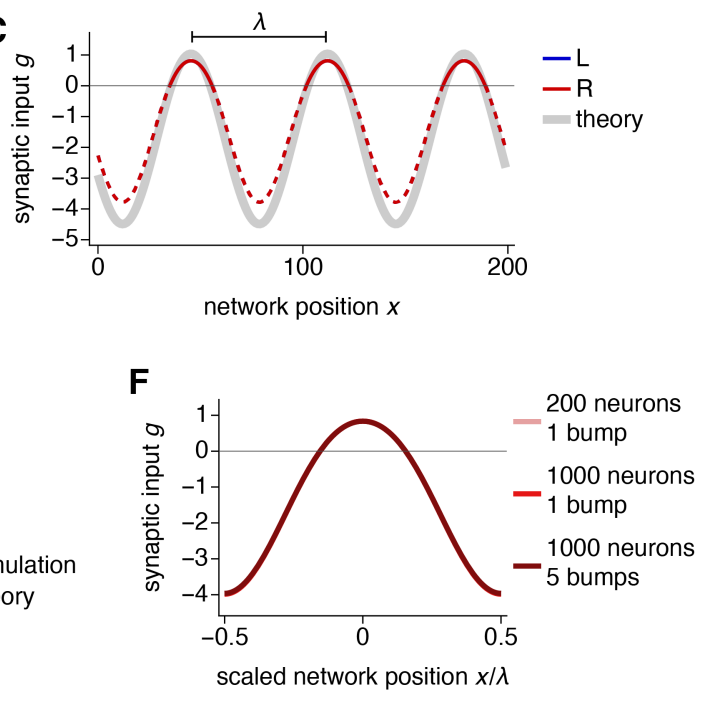

Figure 2: Bump formation in a ring attractor network. (A) Network schematic with populations $L$ and $R$ and locally inhibitory connectivity $W$. (B, C) Networks with 200 neurons and 3 bumps. (B) Connectivity weights for a neuron at the origin. The inhibition distance is $l=29$ and the connectivity shift is $\xi=2$. (C) Steady-state synaptic inputs. Curves for both populations lie over each other. With a ReLU activation function, the firing rates follow the solid portions of the colored lines and are 0 over the dashed portions. The bump distance is $\lambda=200 / 3$. Thick gray line indicates Eq. 4. (D, E) Networks with 500 neurons. (D) More bumps and shorter bump distances are produced by smaller inhibition distances. Points indicate data from 10 replicate simulations. Line indicates Eq. 5. (E) The inhibition distance $l=55$ corresponds to the black point in $\mathbf{D}$ with $\lambda=125$ and $M=4$. These values also minimize the Lyapunov functional (Eq. 6), which varies smoothly across $\lambda$ for infinite networks (line) and takes discrete values for finite networks (points). (F) The scaled bump shape remains invariant across network sizes and bump numbers, accomplished by rescaling connectivity strengths according to Eq. 7. Curves for different parameters lie over one another.

where $\pm_{\mathrm{L}}$ means - and $\pm_{\mathrm{R}}$ means + . Aside from spiking simulations, firing rates $s$ are given by

$$
s_{\alpha}(x, t)=\phi\left[g_{\alpha}(x, t)\right]
$$

where $\phi$ is a nonlinear activation function. For all results in this section, we take $\phi$ to be the rectified linear unit (ReLU) activation function (Eq. 34). $W$ is the synaptic connectivity and only depends on the presynaptic population $\beta$. It obeys a standard continuous attractor architecture based on local inhibition that is strongest at an inhibition distance $l$ (Fig. 2B; Eq. 37). Each population has its synaptic outputs shifted by a small distance $\xi \ll l$ in opposite directions. $A$ is the resting input to all neurons. The driving input, or drive, $b$ is proportional to changes in the coordinate encoded by the network; for the physical coordinates in Fig. 1B, it represents the animal's velocity obtained from self-motion cues. In our results, $b$ is constant in time. It is coupled to the network with strength $\gamma$. We will consider various forms of noise $\zeta$. Finally, $\tau$ is the neural time constant.

With no drive $b=0$ and no noise $\zeta=0$, the network dynamics in Eqs. 1 and 2 can be simplified to

where $2 W(x-y)=\sum_{\beta} W_{\beta}(x, y)$ and the synaptic inputs $g$ are equal between the two populations. This 
baseline equation evolves towards a periodic steady-state $g$ with approximate form (see also Widloski, 2015) 102

$$
g(x)=a \cos \frac{2 \pi\left(x-x_{0}\right)}{\lambda}+d .
$$

Expressions for $a$ and $d$ are given in the Theoretical model section (Eq. 59). The firing rates $s(x)=\phi[g(x)] \quad 103$ exhibit attractor bumps with periodicity $\lambda$, a free parameter that we call the bump distance (Fig. $2 \mathrm{C}$ ). $x_{0}$ is the arbitrary position of one of the bumps. It parameterizes the attractor manifold with each value corresponding to a different attractor state.

The bump number $M=N / \lambda$ is determined through $\lambda$. It can be predicted by the fastest-growing mode in a linearized version of the dynamics (Eq. 42; Sorscher et al. 2019; Khona et al. 2022). The mode with wavenumber $q$ and corresponding wavelength $2 \pi / q$ grows at rate $(2 \tilde{W}(q)-1) / \tau$, where $\tilde{W}(q)$ is the Fourier transform of $W(x)$. Thus,

$$
\frac{2 \pi}{\lambda}=\underset{q}{\operatorname{argmax}} \tilde{W}(q)
$$

Figure 2D shows that simulations follow the predicted $\lambda$ and $M$ over various inhibition distances $l$. Occasionally for small $l$, a different mode with a slightly different wavelength will grow quickly enough to dominate the network. A periodic network enforces an integer bump number, which discretizes the allowable wavelengths and prevents changes in $\lambda$ and $M$ once they are established. In an aperiodic or infinite system, the wavelength can smoothly vary from an initial value to a preferred length over the course of a simulation (Burak and Fiete, 2009; Kang and Balasubramanian, 2019). To determine this preferred $\lambda$ theoretically, we notice that the nonlinear dynamics in Eq. 3 obey the Lyapunov functional

$$
L=-\iint \mathrm{d} x \mathrm{~d} y W(x-y) s(x) s(y)+\int \mathrm{d} x \int_{0}^{s(x)} \mathrm{d} \rho \phi^{-1}[\rho]-A \int \mathrm{d} x s(x)
$$

In the Theoretical model section, we find that $L$ is minimized when $q=2 \pi / \lambda$ maximizes $\tilde{W}(q)$ (Eq. 65 ). This is the same condition as the fastest-growing mode in Eq. 5 (Fig. 2E). In other words, the wavelength $\lambda$ most likely to be established in a periodic network is the preferred bump distance in an aperiodic or infinite system, up to a small difference due to discretization.

We now understand how to produce different bump numbers $M$ in networks of different sizes $N$ by adjusting the inhibition distance $l$. To compare networks across different values of $M$ and $N$, we scale the connectivity strength $W$ according to

$$
W_{\beta}(x, y) \propto \frac{M}{N}
$$

This keeps the total connectivity strength per neuron $\int \mathrm{d} x W_{\beta}(x, y)$ constant over $M$ and $N$. In doing so, the shape of each attractor bump as a function of scaled network position $x / \lambda$ remains invariant (Fig. 2F). Thus,

Eq. 7 isolates our comparisons across $M$ and $N$ to those variables themselves and removes any influence of bump shape.

\section{Bump dynamics: path integration and diffusion}

The drive $b$ produces coherent bump motion by creating an imbalance between the two neural populations. A positive $b$ increases input to the $\mathrm{R}$ population and decreases input to the L population (Fig. 3A). Because 
the synaptic outputs of the former are shifted to the right, the bump moves in that direction. Similarly, a negative $b$ produces leftward bump motion. The bump velocity $v_{\text {drive }}$ can be calculated in terms of the baseline firing rates $s(x)$ obtained without drive and noise (see also Xie et al., 2002; Mosheiff and Burak, 2019):

$$
v_{\text {drive }}=-\frac{\gamma b \xi \int \mathrm{d} x \frac{\mathrm{d}^{2} s}{\mathrm{~d} x^{2}}}{\tau \int \mathrm{d} x\left(\frac{\mathrm{d} s}{\mathrm{~d} x}\right)^{2}} .
$$

As a note, these integrals, as well as subsequent ones, do not include the singular points at the edges of attractor bumps. Equation 8 states that bump velocity is proportional to drive $b$ and connectivity shift $\xi$, which is reflected in our simulations, with some deviation at larger $\xi$ (Fig. 3B, C). The strict proportionality between $v$ and $b$ is crucial because it implies faithful path integration (Burak and Fiete, 2009). If $b(t)$ represents coordinate changes (such as angular or linear velocity in Fig. 1B), then the bump position $\theta(t)$ will accurately track the coordinate itself (orientation or position).

In contrast to drive, uncorrelated noise $\zeta$ produces bump diffusion. To illustrate this effect, we introduce one form of $\zeta$ that we call synaptic input noise. Suppose $\zeta$ is independently sampled for each neuron at each simulation timestep from a Gaussian distribution with mean 0 and variance $\sigma^{2}$. Loosely, it can arise from applying the central limit theorem to the multitude of noisy synaptic and cellular processes occurring at short timescales. Then,

$$
\left\langle\zeta_{\alpha}(x, t)\right\rangle=0, \quad\left\langle\zeta_{\alpha}(x, t) \zeta_{\beta}\left(y, t^{\prime}\right)\right\rangle=\sigma^{2} \Delta t \delta\left(t-t^{\prime}\right) \delta_{\alpha \beta} \delta(x-y)
$$

where the timestep $\Delta t$ sets the resampling rate of $\zeta$, and angle brackets indicate averaging over an ensemble ${ }_{147}$ of replicate simulations. Input noise causes bumps to diffuse away from the coherent driven motion (Fig. 3D). The mean velocity $\langle v\rangle$ remains proportional to drive $b$, which means that the network still path integrates on average (Fig. 3E). Since $\langle v\rangle$ is largely independent of noise magnitude $\sigma$, and the bump diffusion coefficient $D$ is largely independent of $b$, drive and input noise do not significantly interact within the explored parameter range (Fig. 3F, G). D can be calculated in terms of the baseline firing rates (see also Wu et al., 2008; Burak and Fiete, 2012):

$$
D_{\text {input }}=\frac{\sigma^{2} \Delta t}{4 \tau^{2} \int \mathrm{d} x\left(\frac{\mathrm{d} s}{\mathrm{~d} x}\right)^{2}} .
$$

The quadratic dependence of $D$ on $\sigma$ is confirmed by simulation (Fig. 3H).

We now turn our attention to bump number $M$ and network size $N$. The mean bump velocity $\langle v\rangle$ is independent of these parameters (Fig. 3E, F), which can be understood theoretically. Bump shapes across $M$ and $N$ are simple rescalings of one another (Fig. 2F), so derivatives of $s$ with respect to $x$ are simply proportional to $M$ (more bumps imply faster changes) and inversely proportional to $N$ (larger networks imply slower changes). Similarly, integrals of expressions containing $s$ over $x$ are simply proportional to $N$. In summary,

$$
\frac{\mathrm{d} s}{\mathrm{~d} x} \propto \frac{M}{N}, \quad \frac{\mathrm{d}^{2} s}{\mathrm{~d} x^{2}} \propto \frac{M^{2}}{N^{2}}, \quad \int \mathrm{d} x \propto N .
$$

Applying these scalings to Eq. 8, we indeed expect $v_{\text {drive }}$ to be independent of $M$ and $N$. In contrast, ${ }_{161}$ 
A

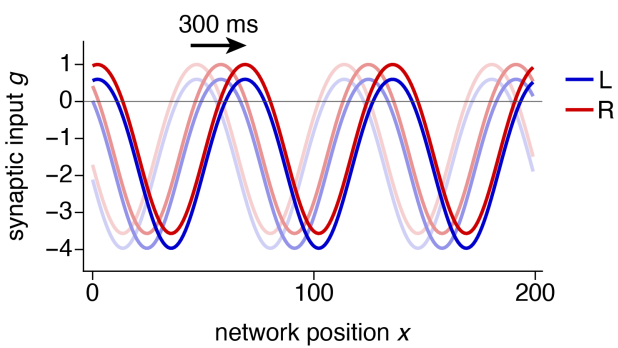

B

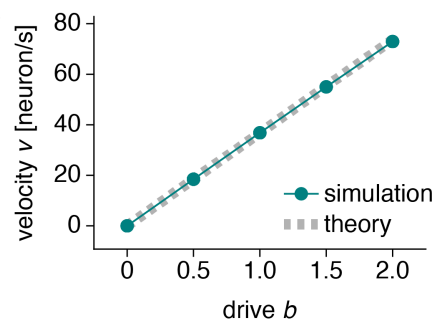

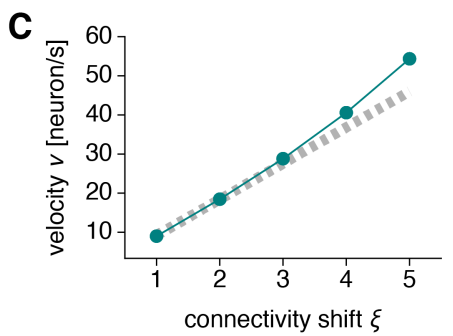

E

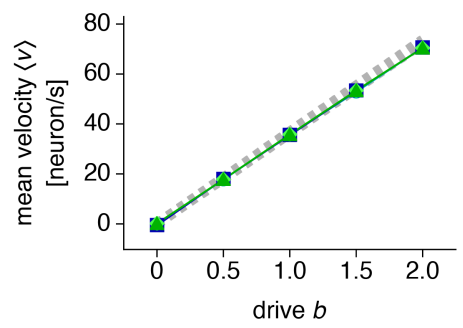

G

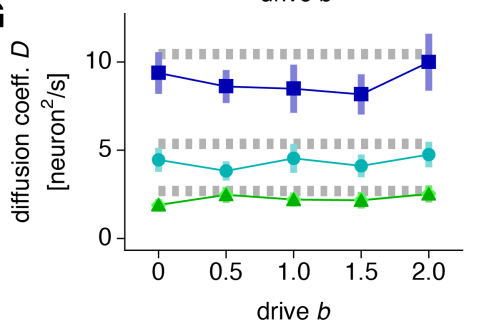

$\mathbf{F}$

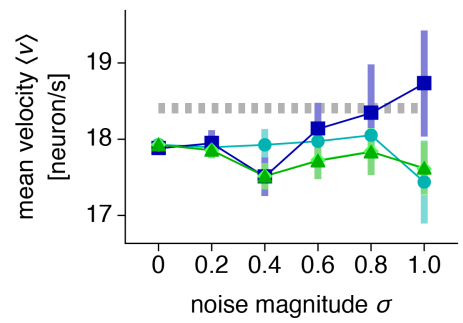

H

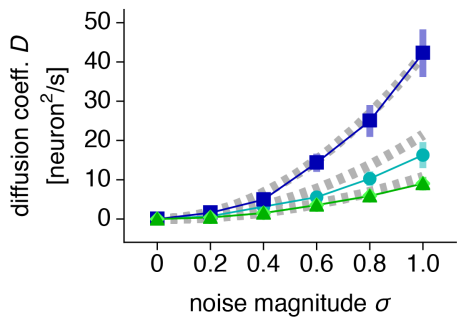

Figure 3: Dynamics in a ring attractor network. (A-C) Networks with 200 neurons and 3 bumps. (A) Synaptic inputs for populations $\mathrm{L}$ and $\mathrm{R}$ under drive $b=2$. Snapshots taken at $150 \mathrm{~ms}$ intervals demonstrate rightward motion. (B) Bump velocity is proportional to drive. The connectivity shift is $\xi=2$. (C) Bump velocity is largely proportional to connectivity shift. The drive is $b=0.5$. (D-H) Networks with synaptic input noise. (D) Bump displacements for 48 replicate simulations demonstrating diffusion with respect to coherent motion. Networks with 200 neurons and 1 bump. $(E, F)$ Mean bump velocity is proportional to drive and remains largely independent of network size, bump number, and noise magnitude. (G, H) Bump diffusion coefficient scales quadratically with noise magnitude, remains largely independent of drive, and varies with network size and bump number. The noise magnitude is $\sigma=0.5 \mathrm{in} \mathbf{D}$, $\mathbf{E}$, and $\mathbf{G}$, and the drive is $b=0.5$ in $\mathbf{D}, \mathbf{F}$, and $\mathbf{H}$. Values for both bumps in two-bump networks lie over each other. Points indicate data from 48 replicate simulations and bars indicate bootstrapped standard deviations. Dotted gray lines indicate Eqs. 8 and 10.

Fig. 3G, $\mathrm{H}$ reveals that the diffusion coefficient $D$ varies with these parameters. When a one-bump network is increased in size from 200 to 400 neurons, $D$ increases as well, which implies greater path integration errors. This undesired effect can be counteracted by increasing the bump number from 1 to 2, which lowers $D$ below that of the one-bump network with 200 neurons. These initial results suggest that bump number and network size are important factors in determining a CAN's resilience to noise. We will explore this idea in greater detail.

\section{Mapping network coordinates onto physical coordinates}

Before further comparing networks with different bump numbers $M$ and sizes $N$, we should scrutinize the relationship between bump motion and the physical coordinate encoded by the network. After all, the latter is typically more important in biological settings. First, we consider the trivial case in which each 
A linear coordinates: 1 neuron $\leftrightarrow 1 \mathrm{~cm}$

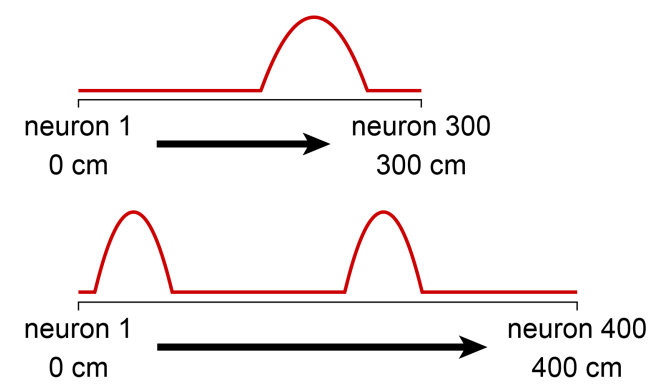

B circular coordinates: 1 bump $\leftrightarrow 360^{\circ}$

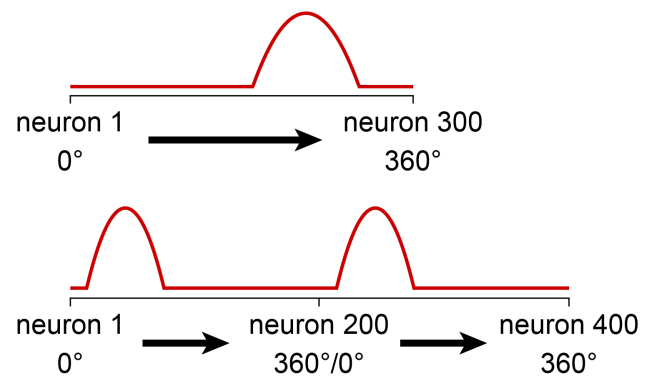

Figure 4: Possible mappings between network coordinates and two types of physical coordinates. (A) In networks encoding linear coordinates such as position, one neuron always represents a fixed physical interval. This mapping is trivial and identical to using network coordinates. (B) In networks encoding circular coordinates such as orientation, the bump distance always represents $360^{\circ}$.

neuron represents a fixed physical interval across all $M$ and $N$; this is equivalent to using network coordinates without a physical mapping (Fig. 4A). It is suited for encoding linear variables like position that lack intrinsic periodicity, so larger networks can encode wider coordinate ranges. We assume that any ambiguity among coordinates encoded by each bump can be resolved by additional cues, such as local features, that identify the true value among the possibilities (O'Keefe and Burgess, 2005; Sreenivasan and Fiete, 2011; Stemmler et al., 2015). We leave quantities with dimensions of network distance in natural units of neurons.

Multi-bump networks are intrinsically periodic, especially those with a ring architecture. A natural way for them to encode a circular coordinate like orientation would be to match network and physical periodicities. For example, the bump distance may always represent $360^{\circ}$ across different $M$ and $N$ so that neurons always exhibit unimodal tuning (Fig. 4B). This relationship implies that quantities with dimensions of network distance should be multiplied by powers of the conversion factor

$$
\frac{360^{\circ} \cdot M}{N}
$$

which converts units of neurons to degrees.

For circular mapping, we must also ensure that networks with different bump numbers $M$ and sizes $N$ path integrate consistently with one another. The same drive $b$ should produce the same bump velocity $v$ in units of degree/s. To do so, we rescale the coupling strength $\gamma$ only under circular mapping:

$$
\gamma \propto \frac{N}{M}
$$

This effectively compensates for the factor of $M / N$ in Eq. 12. To see this explicitly, recall that $v_{\text {drive }}$ does not depend on $M$ and $N$ in units of neuron/s, as shown in Fig. 3E, F and previously explained through scaling arguments. Under circular mapping, $v_{\text {drive }}$ would be multiplied by one power of the conversion factor in Eq. 12. Since its formula contains $\gamma$ in the numerator (Eq. 8), $v_{\text {drive }}$ receives an additional power of the scaling factor in Eq. 13. The two factors cancel each other, so $v_{\text {drive }}$ does not depend on $M$ and $N$ under either mapping:

$$
v_{\text {drive }} \propto 1 \text { linear, } \quad v_{\text {drive }} \propto 1 \text { circular. }
$$




\section{A}
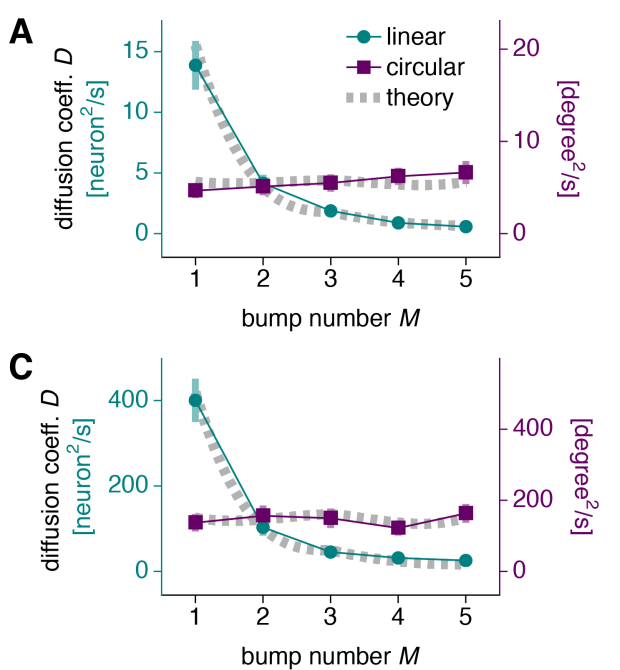

B
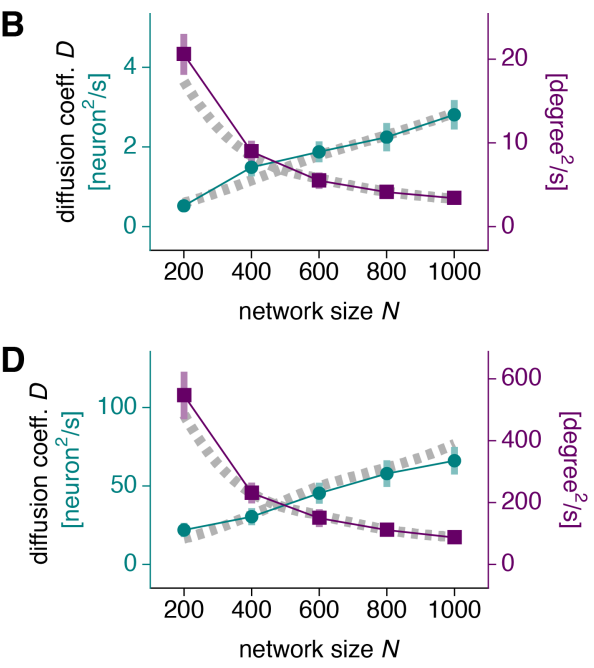

Figure 5: Bump diffusion due to input and spiking noise. (A, B) Networks with synaptic input noise of magnitude $\sigma=0.5$ and drive $b=0.5$. Dotted gray lines indicate Eq. 10. (A) Diffusion decreases with bump number under linear mapping and remains largely constant under circular mapping. Networks with 600 neurons. (B) Diffusion increases with network size under linear mapping and decreases under circular mapping. Networks with 3 bumps. (C, D) Same as A, B, but for networks with Poisson spiking noise instead of input noise. Dotted gray lines indicate Eq. 20. Points indicate data from 48 replicate simulations and bars indicate bootstrapped standard deviations.

Thus, a consistent relationship between $b$ and $v_{\text {drive }}$ is preserved in units of both neurons/s and degrees/s.

Of course, there are other possible mappings between network and physical coordinates across bump numbers and network sizes, but for the rest of our paper, we will consider these two. We will see that noise has different consequences in networks encoding linear and circular coordinates.

\section{More bumps improve robustness to input and spiking noise under linear mapping}

We now revisit the effect of input noise on bump diffusion, as explored in Fig. 3D-H. We measure how the 198 diffusion coefficient $D$ varies with bump number $M$ and network size $N$ under linear and circular mappings. Under linear mapping, $D$ decreases as a function of $M$ but increases as a function of $N$ (Fig. 5A-B). Thus, more bumps attenuate diffusion produced by input noise, which is especially prominent in large networks. However, for circular coordinates, $D$ remains largely constant with respect to $M$ and decreases with respect to $N$ (Fig. 5A-B). Increasing the number of bumps provides no benefit. These results can be understood through Eqs. 10 to 12, which predict

$$
D_{\text {input }} \propto \frac{N}{M^{2}} \text { linear, } \quad D_{\text {input }} \propto \frac{1}{N} \quad \text { circular. }
$$

Two powers of the conversion factor in Eq. 12 account for the differences between the two mappings.

Next, we investigate networks with spiking noise instead of input noise. To do so, we replace the deterministic formula for firing rate in Eq. 2 with

$$
s_{\alpha}(x, t)=\frac{c_{\alpha}(x, t)}{\Delta t}
$$


Here, $s$ is a stochastic, instantaneous firing rate given by the number of spikes $c$ emitted in a simulation 208 timestep divided by the timestep duration $\Delta t$. We take the $c$ 's to be independent Poisson random variables 209 driven by the deterministic firing rate:

$$
c_{\alpha}(x, t) \sim \operatorname{Pois}\left[\phi\left[g_{\alpha}(x, t)\right] \Delta t\right]
$$

As fully explained in the Theoretical model section (Eq. 98), we can approximate this spiking process by the rate-based dynamics in Eqs. 1 and 2 with the noise term

$$
\zeta_{\alpha}(x, t)=\sum_{\beta} \int \mathrm{d} y W_{\beta}(x, y) \sqrt{\frac{\phi\left[g_{\beta}(y, t)\right]}{\Delta t}} \eta_{\beta}(y, t) .
$$

The $\eta$ 's are independent random variables with zero mean and unit variance:

$$
\left\langle\eta_{\alpha}(x, t)\right\rangle=0, \quad\left\langle\eta_{\alpha}(x, t) \eta_{\beta}\left(y, t^{\prime}\right)\right\rangle=\Delta t \delta\left(t-t^{\prime}\right) \delta_{\alpha \beta} \delta(x-y)
$$

As for Eq. 9, the simulation timestep $\Delta t$ sets the rate at which $\eta$ is resampled. This spiking noise produces bump diffusion with coefficient (see also Burak and Fiete, 2012)

$$
D_{\text {spike }}=\frac{\int \mathrm{d} x s(x)\left(\frac{\mathrm{d} s}{\mathrm{~d} x}\right)^{2}}{4 \tau^{2}\left[\int \mathrm{d} x\left(\frac{\mathrm{d} s}{\mathrm{~d} x}\right)^{2}\right]^{2}}
$$

As before, $s$ is the baseline firing rate configuration without noise and drive. Through the relationships in 216

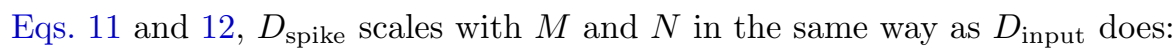

$$
D_{\text {spike }} \propto \frac{N}{M^{2}} \text { linear, } \quad D_{\text {spike }} \propto \frac{1}{N} \quad \text { circular. }
$$

These findings are presented in Fig. 5C-D along with simulation results that confirm our theory. Spiking noise behaves similarly to input noise. Increasing bump number improves robustness to noise under linear mapping but has almost no effect under circular mapping. Bump diffusion in larger networks is exacerbated under linear mapping but suppressed under circular mapping.

\section{More bumps improve robustness to connectivity noise under linear mapping}

Another source of noise in biological CANs is inhomogeneity in the connectivity $W$. Perfect continuous attractor dynamics requires $W$ to be invariant to translations along the network (Skaggs et al., 1995; Zhang, 1996; Samsonovich and McNaughton, 1997; Fuhs and Touretzky, 2006; Burak and Fiete, 2009), a concept related to Goldstone's theorem in physics (Nambu, 1960; Goldstone, 1961). We consider the effect of replacing $W \rightarrow W+V$, where $V$ is a noisy connectivity matrix whose entries are independently drawn from a zeromean Gaussian distribution. $V$ disrupts the symmetries of $W$. This noise is quenched and does not change over the course of the simulation, in contrast to input and spiking noise which are independently sampled in 

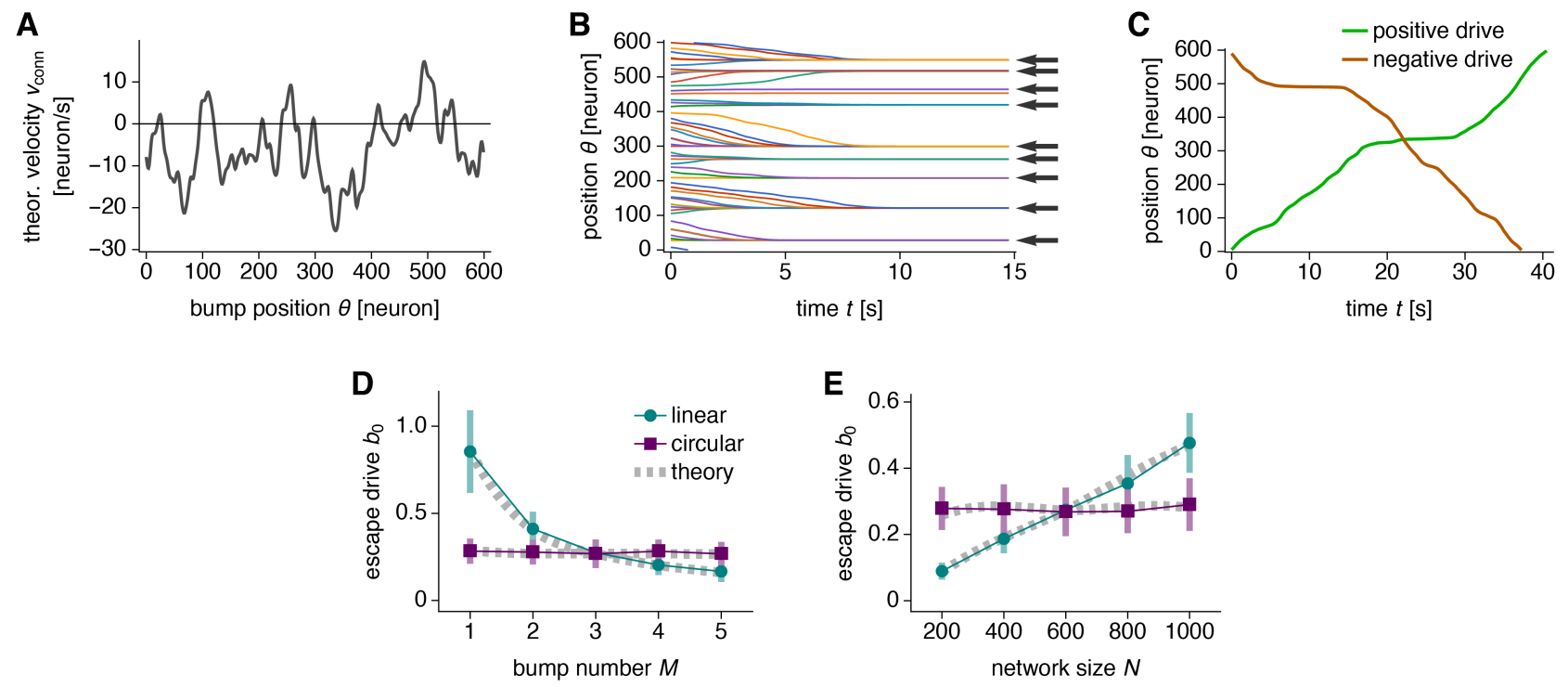

Figure 6: Bump trapping due to connectivity noise at low drive. (A-C) Networks with 600 neurons, 1 bump, and the same realization of connectivity noise of magnitude 0.002 . (A) Theoretical values for drift velocity as a function of bump position using Eq. 23. (B) Bumps drift towards trapped positions over time. The drive is $b=0$. Arrows indicate predictions from $v_{\text {conn }}(\theta)$ crossing 0 with negative slope in $\mathbf{A}$. Lines indicate simulations with different starting positions. (C) Bump trajectories with smallest positive and negative drive required to travel through the entire network. Respectively, $b=0.75$ and $b=-0.52$. The larger of the two in magnitude is the escape drive $b_{0}=0.75$. Note that positions with low bump speed exhibit large velocities in the opposite direction in A. (D-E) Networks with multiple realizations of connectivity noise of magnitude 0.002 . (D) Escape drive decreases with bump number under linear mapping and remains largely constant under circular mapping. Networks with 600 neurons. (E) Escape drive increases with network size under linear mapping and remains largely constant under circular mapping. Networks with 3 bumps. Points indicate simulation means over 48 realizations and bars indicate standard deviations. Dotted gray lines indicate Eq. 25 averaged over 96 realizations.

time. $V$ creates a noise term

$$
\zeta_{\alpha}(x, t)=\sum_{\beta} \int \mathrm{d} y V_{\alpha \beta}(x, y) s_{\beta}(y, t)
$$

Equation 22 shows that $V$ produces correlated $\zeta$ 's across neurons, which also differs from input and spiking noise. Because of these distinctions, the dominant effect of connectivity noise is not diffusion, but drift. $V$ induces bumps to move with velocity $v_{\text {conn }}(\theta)$, even without drive $b$ :

$$
v_{\text {conn }}(\theta)=-\frac{\sum_{\alpha \beta} \iint \mathrm{d} x \mathrm{~d} y V_{\alpha \beta}(x, y) \frac{\mathrm{d} s(x-\theta)}{\mathrm{d} x} s(y-\theta)}{2 \tau \int \mathrm{d} x\left(\frac{\mathrm{d} s}{\mathrm{~d} x}\right)^{2}} .
$$

The movement is coherent but irregular, as it depends on the bump position $\theta$ (Fig. 6A). Itskov et al. (2011) ${ }_{234}$ and Seeholzer et al. (2019) refer to $v_{\text {conn }}(\theta)$ as the drift velocity.

Connectivity noise traps bumps at low drive $b$. We first consider $b=0$, so bump motion is governed solely by drift according to $\mathrm{d} \theta / \mathrm{d} t=v_{\text {conn }}(\theta)$. The bump position $\theta$ has stable fixed points wherever $v_{\text {conn }}(\theta)$ crosses 0 with negative slope (Itskov et al., 2011; Seeholzer et al., 2019). Simulations confirm that bumps 
drift towards these points (Fig. 6B). The introduction of $b$ adds a constant $v_{\text {drive }}$ that moves the curve in 239 Fig. 6A up for positive $b$ or down for negative $b$ :

$$
v_{\text {total }}(\theta)=v_{\text {drive }}+v_{\text {conn }}(\theta)
$$

If $v_{\text {total }}(\theta)$ still crosses 0 , bumps would still be trapped. The absence of bump motion in response to coordinate changes encoded by $b$ would be a catastrophic failure of path integration. To permit bump motion through the entire network, the drive must be strong enough to eliminate all zero-crossings. Figure $6 \mathrm{C}$ shows bump motion at this drive for both directions of motion. The positive $b$ is just large enough for the bump to pass through the region with the most negative $v_{\text {conn }}(\theta)$ in Fig. 6A; likewise for negative $b$ and positive $v_{\text {conn }}(\theta)$. We call the larger absolute value of these two drives the escape drive $b_{0}$. Simulations show that $b_{0}$ decreases with bump number $M$ and increases with network size $N$ under linear mapping (Fig. 6D, E). A smaller $b_{0}$ implies weaker trapping, so smaller networks with more bumps are more resilient against this phenomenon. Under circular mapping, however, $b_{0}$ demonstrates no significant dependence on $M$ or $N$. We can predict $b_{0}$ by inverting the relationship in Eq. 8 between $b$ and $v$ :

$$
b_{0}=-\frac{\max _{\theta}\left|v_{\operatorname{conn}}(\theta)\right| \cdot \tau \int \mathrm{d} x\left(\frac{\mathrm{d} s}{\mathrm{~d} x}\right)^{2}}{\gamma \xi \int \mathrm{d} x \frac{\mathrm{d}^{2} s}{\mathrm{~d} x^{2}}} .
$$

This theoretical result agrees well with values obtained by simulation (Fig. 6D, E). In the Theoretical model section, we present a heuristic argument (Eq. 123) that leads to the observed scaling of escape drive on $M$ and $N$ :

$$
b_{0} \propto \frac{N}{M} \quad \text { linear, } \quad b_{0} \propto 1 \quad \text { circular. }
$$

At high drive $|b|>b_{0}$, attractor bumps are no longer trapped by the drift velocity $v_{\text {conn }}(\theta)$. Instead, the drift term produces irregularities in the total velocity $v_{\text {total }}(\theta)$ (Fig. 7A). They can be decomposed into two components: irregularities between directions of motion and irregularities within each direction. Both imply errors in path integration because $v$ and $b$ are not strictly proportional. To quantify these components, we call $\left|v_{+}(\theta)\right|$ and $\left|v_{-}(\theta)\right|$ the observed bump speeds under positive and negative $b$. We define speed difference as the unsigned difference between mean speeds in either direction, normalized by the overall mean speed:

$$
\text { speed difference }=\frac{2\left|\operatorname{mean}_{\theta}\right| v_{+}(\theta)\left|-\operatorname{mean}_{\theta}\right| v_{-}(\theta)||}{\operatorname{mean}_{\theta}\left|v_{+}(\theta)\right|+\underset{\theta}{\operatorname{mean}}\left|v_{-}(\theta)\right|} .
$$

We then define speed variability as the standard deviation of speeds within each direction, averaged over 260 both directions and normalized by the overall mean speed:

$$
\text { speed variability }=\frac{\operatorname{std}_{\theta}\left|v_{+}(\theta)\right|+\operatorname{std}_{\theta}\left|v_{-}(\theta)\right|}{\operatorname{mean}_{\theta}\left|v_{+}(\theta)\right|+\operatorname{mean}_{\theta}\left|v_{-}(\theta)\right|} .
$$

Speed difference and speed variability follow the same trends under changes in bump number $M$ and network ${ }_{262}$ size $N$ (Fig. 7B-E). Under linear mapping, they decrease with $M$ and increase with $N$. Under circular ${ }_{263}$ 


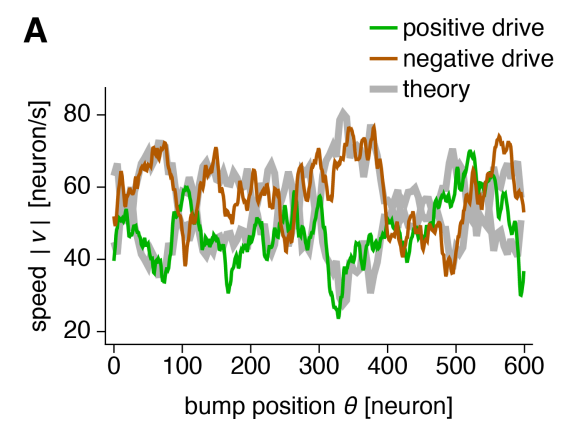

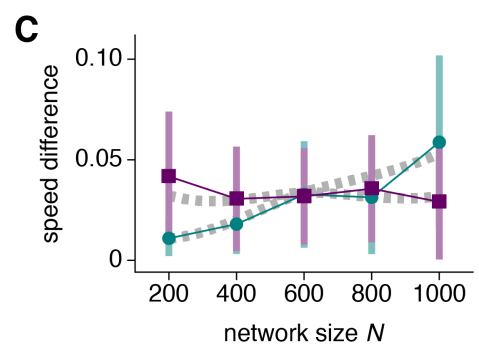
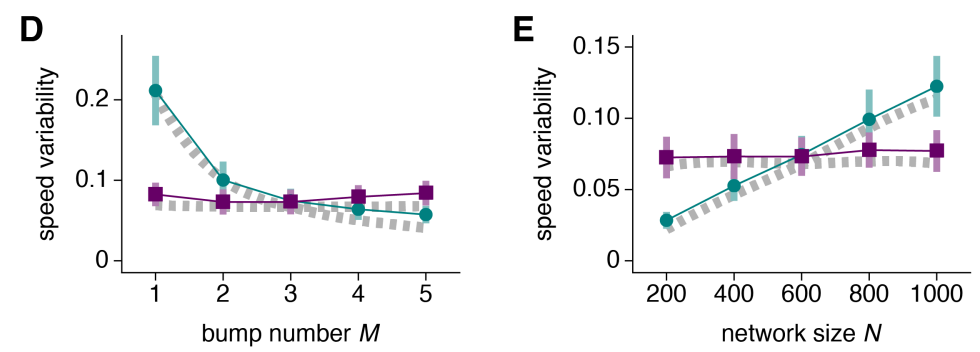

Figure 7: Bump speed irregularity due to connectivity noise at high drive. (A) Bump speed as a function of bump position with connectivity noise of magnitude 0.002 and drive $b=1.5$. Network with 600 neurons, 1 bump, and the same realization of connectivity noise as in Fig. 5A-C. Thick gray lines indicate Eq. 24. (B-E) Networks with multiple realizations of connectivity noise of magnitude 0.002 and drive $b=1.5$. (B) Speed difference between directions decreases with bump number under linear mapping and remains largely constant under circular mapping. Networks with 600 neurons. (C) Speed difference increases with network size under linear mapping and remains largely constant under circular mapping. Networks with 3 bumps. (D, E) Same as B, C, but for speed variability within each direction. Points indicate simulation means over 48 realizations and bars indicate standard deviations. Dotted gray lines indicate Eqs. 29 and 30 averaged over 96 realizations.

mapping, they do not significantly depend on $M$ and $N$. These are also the same trends exhibited by ${ }_{264}$ the escape drive $b_{0}$ (Fig. 6D, E). In terms of theoretical quantities, the formulas for speed difference and 265 variability become

$$
\text { speed difference }=\frac{2\left|\operatorname{mean}_{\theta} v_{\text {conn }}(\theta)\right|}{\left|v_{\text {drive }}\right|}
$$

and

$$
\text { speed variability }=\frac{\operatorname{std} v_{\text {conn }}(\theta)}{\left|v_{\text {drive }}\right|} .
$$

These expressions match the observed values well (Fig. 7B-E). In the Theoretical methods section, we calculate the observed dependence of speed difference (Eq. 112) and variability (Eq. 119) on $M$ and $N$ :

$$
\text { speed difference and variability } \propto \frac{N}{M} \text { linear, } \quad \text { speed difference and variability } \propto 1 \text { circular. }
$$

To summarize, CANs with imperfect connectivity benefit from more attractor bumps when encoding linear coordinates. This advantage is present at all driving inputs and may be more crucial for larger networks. On the other hand, connectivity noise has largely the same consequences for networks of all bump numbers and sizes when encoding circular coordinates. 


\section{Discussion}

We demonstrated how CANs capable of path integration respond to three types of noise. Additive synaptic input noise and Poisson spiking noise cause bumps to diffuse away from the coherent motion responsible for path integration (Figs. 3 and 5). Connectivity noise produces a drift velocity field that also impairs path integration by trapping bumps at low drive and perturbing bump motion at high drive (Figs. 6 and 7).

For all three types of noise, CANs with more attractor bumps exhibit less deviation in bump motion in network units. Thus, they can more robustly encode linear variables whose mapping inherits these units and does not rescale with bump number (Fig. 4A). If grid cell networks were to encode spatial position in this manner, then multiple attractor bumps would be preferred over a single bump. Additional bumps introduce greater ambiguity among positions encoded by each bump, but this can be resolved by a rough estimate of position from additional cues, such as local landmarks (O'Keefe and Burgess, 2005; Krupic et al., 2014; Bush and Burgess, 2014; Hardcastle et al., 2015), another grid module with different periodicity (O'Keefe and Burgess, 2005; McNaughton et al., 2006; Stensola et al., 2012; Stemmler et al., 2015; Kang and Balasubramanian, 2019; Khona et al., 2022), or a Bayesian prior based on recent memory (Sreenivasan and Fiete, 2011). In this way, grid modules with finer resolution and more attractor bumps could maintain a precise egocentric encoding of position, while coarser modules and occasional allocentric cues would identify the true position out of the few possibilities represented. Gu et al. (2018) report experimental evidence supporting multi-bump grid networks obtained by calcium imaging of mouse medial entorhinal cortex. Our work implies that the evolution of such networks may have been partially encouraged by biological noise. In contrast, CANs encoding circular variables may rescale under different bump numbers to match periodicities (Fig. 4B), which eliminates any influence of bump number on encoding accuracy for all three types of noise. If head direction networks were to encode orientation in this manner, then they would face less selective pressure to evolve beyond the single-bump configuration observed in Drosophila (Seelig and Jayaraman, 2015). Further experimental characterization of bump number in biological CANs, perhaps through techniques proposed by Widloski et al. (2018), would test the degree to which the brain can leverage the theoretical advantages identified in this work.

Under linear mapping, larger CANs exhibit more errors in path integration from all three types of noise. The immediate biological implication is that larger brains face a dramatic degradation of CAN performance, accentuating the importance of suppressing error with multi-bump networks. However, this simple rule that one neuron always represents a fixed physical interval does not need to be followed. Furthermore, larger animals may tolerate greater absolute errors in path integration because they interact with their environments over larger scales. Nevertheless, our results highlight the importance of considering network size when studying the performance of noisy CANs. Under circular mapping, bump diffusion decreases with network size for input and spiking noise, and the magnitude of errors due to connectivity noise is independent of network size. This implies that head direction networks can benefit from incorporating more neurons; the observed interactions between such networks across different mammalian brain regions may act in this manner to suppress noise (Taube, 2007).

The computational advantages of periodic over nonperiodic encodings has been extensively studied in the context of grid cells (Fiete et al., 2008; Sreenivasan and Fiete, 2011; Mathis et al., 2012; Wei et al., 2015; Stemmler et al., 2015). Our results enhance these findings by demonstrating that some kinds of periodic encodings can perform better than others. Our results also contribute to a rich literature on noisy CANs. 
Previous studies have investigated additive input noise (Compte et al., 2000; Wu et al., 2008; Burak and 315 Fiete, 2012; Kilpatrick and Ermentrout, 2013; Seeholzer et al., 2019), spiking noise (Burak and Fiete, 2009, 316 2012; Seeholzer et al., 2019), and quenched noise due to connectivity or input inhomogeneities (Zhang, 1996; Itskov et al., 2011; Kilpatrick and Ermentrout, 2013; Seeholzer et al., 2019). Only Burak and Fiete (2009) consider networks with multiple activity bumps, and they did not study the effects of changing bump number. The novel relationship described here between bump number and robustness to noise has broad significance towards understanding CANs, especially in a biological setting.

Beyond our concrete results on CAN performance, our work offers a comprehensive theoretical framework for understanding CANs. We discover a formula for a multi-bump attractor state and a Lyapunov function for the network dynamics. We calculate all key dynamical quantities such as velocities and diffusion coefficients in terms of firing rates. Our formulas yield scaling relationships that facilitate an intuitive understanding for their dependence on bump number and network size. Much of our theoretical development does not assume a specific connectivity matrix or nonlinear activation function, which allows our results to have wide significance. For example, we expect them to hold for networks with excitatory synapses. Other theories have been developed for bump shape (Wu et al., 2002; Xie et al., 2002; Itskov et al., 2011; Kilpatrick and Ermentrout, 2013; Widloski, 2015), path integration velocity (Xie et al., 2002; Mosheiff and Burak, 2019), diffusion coefficients (Wu et al., 2008; Burak and Fiete, 2012; Kilpatrick and Ermentrout, 2013; Seeholzer et al., 2019), and drift velocity (Zhang, 1996; Itskov et al., 2011; Seeholzer et al., 2019). Our work unifies these studies through a simple framework that features path integration, multiple bumps, and a noise term that can represent a wide range of sources. It can be easily extended to include other components of theoretical or biological significance, such as slowly-varying inputs (Tsodyks and Sejnowski, 1995; Fung et al., 2010; Kilpatrick and Ermentrout, 2013), synaptic plasticity (Stringer et al., 2002; Renart et al., 2003), neural oscillations (Thurley et al., 2008; Navratilova et al., 2012; Kang and DeWeese, 2019), and higher-dimensional attractor manifolds (Ermentrout and Cowan, 1979; Samsonovich and McNaughton, 1997).

\section{Theoretical model}

\section{Architecture}

We investigate CAN dynamics through a 1D ring attractor network. This class of network has been analyzed in previous theoretical works, and at various points, our calculations will parallel those Xie et al. (2002); Itskov et al. (2011); Burak and Fiete (2012); Kilpatrick and Ermentrout (2013); Widloski (2015); Seeholzer et al. (2019); Mosheiff and Burak (2019).

There are two neurons at each position $i=0, \ldots, N-1$ with population indexed by $\alpha \in\{\mathrm{L}, \mathrm{R}\}$ (Fig. 1A). For convenient calculation, we unwrap the ring and connect copies end-to-end, forming a linear network with continuous positions $x \in(-\infty, \infty)$. Unless otherwise specified, integrals are performed over the entire range. To map back onto the finite-sized ring network, we enforce our results to have a periodicity $\lambda$ that divides $N$. Integrals would then be performed over $[0, N)$, with positions outside this range corresponding to their equivalents within this range.

The network obeys the following dynamics for synaptic inputs $g$ :

$$
\tau \frac{\mathrm{d} g_{\alpha}(x, t)}{\mathrm{d} t}+g_{\alpha}(x, t)=\sum_{\beta} \int \mathrm{d} y W_{\beta}(x, y) s_{\beta}(y, t)+A \pm_{\alpha} \gamma b(t)+\zeta_{\alpha}(x, t)
$$

where $\pm_{\mathrm{L}}$ means - and $\pm_{\mathrm{R}}$ means + , and the opposite for $\mp_{\alpha} . \tau$ is the neural time constant, $W$ is the ${ }_{352}$ 
synaptic connectivity, and $A$ is the resting input. The nonlinear activation function $\phi$ converts synaptic inputs to firing rates:

$$
s_{\alpha}(x, t)=\phi\left[g_{\alpha}(x, t)\right]
$$

Most of our results will apply to general $\phi$, but we also consider a ReLU activation function specifically:

$$
\phi[g]= \begin{cases}g & g>0 \\ 0 & g \leq 0\end{cases}
$$

$b$ is the driving input, $\gamma$ is its coupling strength, and $\zeta$ is the noise, which can take different forms. $\gamma b$ and $\zeta$ are small compared to the rest of the right-hand side of Eq. 32. For notational convenience, we will often suppress dependence on $t$.

$W_{\beta}(x, y)$ obeys a standard continuous attractor architecture based on a symmetric and translation invariant $W$ :

$$
W_{\beta}(x, y)=W\left(x-y \mp_{\beta} \xi\right) \quad \text { where } \quad W(-x)=W(x) .
$$

Each population $\beta$ deviates from $W$ by a small shift $\xi \ll N$ in synaptic outputs. Thus, the following ${ }^{361}$ approximation holds:

$$
\sum_{\beta} W_{\beta}(x, y) \approx 2 W(x-y)
$$

We will consider the specific form of $W$ (Fig. 1B):

$$
W(x)=\left\{\begin{array}{ll}
w \cdot \frac{\cos \pi x / l-1}{2} & |x|<2 l \\
0 & |x| \geq 2 l
\end{array}= \begin{cases}w \cdot \frac{\cos k x-1}{2} & |x|<2 \pi / k \\
0 & |x| \geq 2 \pi / k\end{cases}\right.
$$

where $k=\pi / l$.

\section{Baseline configuration without drive and noise}

\section{Linearized dynamics and bump distance $\lambda$}

First, we consider the case of no drive $b=0$ and no noise $\zeta=0$. The dynamical equation Eq. 32 becomes

$$
\tau \frac{\mathrm{d} g_{\alpha}(x)}{\mathrm{d} t}+g_{\alpha}(x)=\sum_{\beta} \int \mathrm{d} y W_{\beta}(x, y) \phi\left[g_{\beta}(y)\right]+A .
$$

Since the right-hand side no longer depends on $\alpha, g$ must be the same for both populations, and we can use 367 Eq. 36 to obtain

$$
\tau \frac{\mathrm{d} g(x)}{\mathrm{d} t}+g(x)=2 \int \mathrm{d} y W(x-y) \phi[g(y)]+A .
$$
$h$, is

We analyze these dynamics using the Fourier transform $\mathcal{F}$. Our chosen convention, applied to the function

$$
\begin{gathered}
\tilde{h}(q)=\mathcal{F}[h](q)=\int \mathrm{d} x \mathrm{e}^{-\mathrm{i} q x} h(x) \\
h(x)=\mathcal{F}^{-1}[\tilde{h}](x)=\int \frac{\mathrm{d} q}{2 \pi} \mathrm{e}^{\mathrm{i} q x} \tilde{h}(q) .
\end{gathered}
$$

Fourier modes $\tilde{h}(q)$ represent sinusoids with wavenumber $q$ and corresponding wavelength $2 \pi / q$. Applying 369 
this transform to Eq. 39, we obtain

$$
\tau \frac{\mathrm{d} \tilde{g}(q)}{\mathrm{d} t}+\tilde{g}(q)=2 \tilde{W}(q) \mathcal{F}[\phi[g]](q)+2 \pi A \delta(q) .
$$

In this subsection, we consider the case of small deviations, such that $g(x) \approx g_{0}$ and $\phi[g(x)] \approx \phi\left[g_{0}\right]+{ }_{371}$ $\phi^{\prime}\left[g_{0}\right]\left(g(x)-g_{0}\right)$. Then, Eq. 41 becomes

$$
\tau \frac{\mathrm{d} \tilde{g}(q)}{\mathrm{d} t}+\tilde{g}(q)=2 \phi^{\prime}\left[g_{0}\right] \tilde{W}(q) \tilde{g}(q)+2 \pi A_{0} \delta(q),
$$

where $A_{0}=A+2 \tilde{W}(0)\left(\phi\left[g_{0}\right]-\phi^{\prime}\left[g_{0}\right] g_{0}\right)$. The solution to this linearized equation for $q \neq 0$ is

$$
\tilde{g}(q, t)=\tilde{g}(q, 0) \mathrm{e}^{r(q) t}
$$

Each mode grows exponentially with rate

$$
r(q)=\frac{2 \phi^{\prime}\left[g_{0}\right] \tilde{W}(q)-1}{\tau}
$$

so the fastest-growing component of $g$ is the one that maximizes $\tilde{W}(q)$, as stated in Eq. 5 of the Results section. The wavelength $2 \pi / q$ of that component predicts the bump distance $\lambda$.

For the specific $W$ in Eq. 37, its Fourier transform is

$$
\tilde{W}(q)=-w \frac{k^{2} \sin \frac{2 \pi q}{k}}{k^{2} q-q^{3}}
$$

so

$$
\lambda=\frac{2 \pi}{\underset{q}{\operatorname{argmin}} \frac{k^{2} \sin \frac{2 \pi q}{k}}{k^{2} q-q^{3}}}=\frac{2 l}{\underset{\psi}{\operatorname{argmin}} \frac{\sin 2 \pi \psi}{\psi-\psi^{3}}} \approx 2.28 l .
$$

$\lambda$ is proportional to $l$, as also noted by Fuhs and Touretzky (2006); Burak and Fiete (2009); Kang and Balasubramanian (2019); Khona et al. (2022).

\section{Bump shape $g$}

We call the steady-state synaptic inputs $g$ without drive and noise the baseline configuration. To calculate its shape, we must account for the nonlinearity of the activation function $\phi$ and return to Eq. 41. We invoke our particular form of $\phi$ in Eq. 34 to calculate $\mathcal{F}[\phi[g]](q) . g$ must be periodic, and its periodicity is the bump distance $\lambda$ with wavenumber $\kappa=2 \pi / \lambda$. Without loss of generality, we take $g$ to have a bump centered at 0 . Since $W$ is symmetric, $g$ is an even function. We define $z$ as the position where $g$ crosses 0 :

$$
g(z)=0
$$

If $g$ is approximately sinusoidal, then $g(x)>0$ wherever $n \lambda-z<x<n \lambda+z$ for any integer $n$. The ReLU activation function in Eq. 34 implies

$$
\phi[g(x)]=g(x) \Phi(x) \quad \text { where } \quad \Phi(x)=\sum_{n=-\infty}^{\infty} \Theta[x-n \lambda+z] \Theta[-(x-n \lambda-z)] .
$$


$\Theta$ is the Heaviside step function. The Fourier transform for $\Phi$ is

$$
\tilde{\Phi}(q)=2 \frac{\sin q z}{q} \sum_{n=-\infty}^{\infty} \mathrm{e}^{-2 \pi \mathrm{i} n q / \kappa}=2 \frac{\sin q z}{q} \sum_{n=-\infty}^{\infty} \delta\left(n-\frac{q}{\kappa}\right)=2 \kappa \frac{\sin q z}{q} \sum_{n=-\infty}^{\infty} \delta(q-n \kappa),
$$

where the second equality comes from the Fourier series for a Dirac comb. Therefore,

$$
\mathcal{F}[\phi[g]](q)=\frac{1}{2 \pi} \int \mathrm{d} q^{\prime} \tilde{\Phi}\left(q-q^{\prime}\right) \tilde{g}\left(q^{\prime}\right)=\frac{1}{\pi} \sum_{n=-\infty}^{\infty} \frac{\sin n \kappa z}{n} \tilde{g}(q-n \kappa),
$$

so Eq. 41 becomes

$$
\tau \frac{\mathrm{d} \tilde{g}(q)}{\mathrm{d} t}+\tilde{g}(q)=\frac{2}{\pi} \tilde{W}(q) \sum_{n=-\infty}^{\infty} \frac{\sin n \kappa z}{n} \tilde{g}(q-n \kappa)+2 \pi A \delta(q)
$$

This equation describes the full dynamics of $g$ with a ReLU activation function. It contains couplings between all modes $q$ that are multiples of the wavenumber $\kappa$, which corresponds to the bump distance.

To find the baseline $g$, we set $\mathrm{d} \tilde{g} / \mathrm{d} t=0$. We also simplify $\tilde{g}(q)$ by only considering the lowest modes that couple to each other: $q=0, \pm \kappa$. Due to symmetry, $\tilde{W}(-q)=\tilde{W}(q)$ and $\tilde{g}(-q)=\tilde{g}(q)$. Eq. 51 gives

$$
\begin{aligned}
& \tilde{g}(0)=\frac{2}{\pi} \tilde{W}(0)[\kappa z \tilde{g}(0)+2 \sin (\kappa z) \tilde{g}(\kappa)]+2 \pi A \delta(0) \\
& \tilde{g}(\kappa)=\frac{2}{\pi} \tilde{W}(\kappa)\left[\sin (\kappa z) \tilde{g}(0)+\left(\kappa z+\frac{\sin 2 \kappa z}{2}\right) \tilde{g}(\kappa)\right] .
\end{aligned}
$$

Now we need to impose Eq. 47: $g(z)=0$. To do so, we note that $\tilde{g}(0)$ and $\tilde{g}(\kappa)$ are both proportional to 393 $\delta(0)$ according to Eq. 52. That means $\tilde{g}(q)$ has the form

$$
\tilde{g}(q)=G_{0} \delta(q)+G \delta(q-\kappa)+G \delta(q+\kappa),
$$

where $G_{0}$ and $G$ are the Fourier modes with delta functions separated. This implies

and $g(z)=0$ implies

$$
g(x)=\frac{G_{0}}{2 \pi}+\frac{G}{\pi} \cos \kappa x
$$

$$
G_{0}=-2 \cos (\kappa z) G
$$

Substituting Eqs. 53 and 55 into Eq. 52, we obtain

$$
\begin{aligned}
\frac{G}{\pi} & =-\frac{\pi A}{2 \tilde{W}(0)(\sin \kappa z-\kappa z \cos \kappa z)+\pi \cos \kappa z} \\
\kappa z-\cos \kappa z \sin \kappa z & =\frac{\pi}{2 \tilde{W}(\kappa)} .
\end{aligned}
$$

We can solve the second equation of Eq. 56 for $\kappa z$ and then substitute it into the first equation to obtain $G$. ${ }_{397}$ This then gives us $g(x)$, which becomes through Eqs. 54 and 55

$$
g(x)=\frac{G}{\pi}(\cos \kappa x-\cos \kappa z)
$$

In particular, let's use the $W$ defined by Eq. 37 with Fourier transform Eq. 45. Then,

$$
\tilde{W}(0)=-\frac{2 \pi w}{k} \quad \text { and } \quad \tilde{W}(\kappa)=-\frac{w}{\kappa} \frac{k^{2}}{k^{2}-\kappa^{2}} \sin \frac{2 \pi \kappa}{k} .
$$


Thus,

$$
\begin{aligned}
g(x) & =\frac{G}{\pi}(\cos \kappa x-\cos \kappa z) \\
\kappa z-\cos \kappa z \sin \kappa z & =-\pi /\left[\frac{2 w}{\kappa} \frac{k^{2}}{k^{2}-\kappa^{2}} \sin \frac{2 \pi \kappa}{k}\right] \\
\frac{G}{\pi} & =A /\left[\frac{4 w}{k}(\sin \kappa z-\kappa z \cos \kappa z)-\cos \kappa z\right] .
\end{aligned}
$$

This provides expressions for $a$ and $d$ in Eq. 4 of the Results section, where $a=G / \pi$ and $d=-(G / \pi) \cos \kappa z . \quad 400$

\section{Lyapunov functional and bump distance $\lambda$}

The dynamical equation in Eq. 39 admits a Lyapunov functional. In analogy to the continuous Hopfield model (Hopfield, 1984), we can define a Lyapunov functional in terms of $s(x)=\phi[g(x)]$ :

$$
L=-\iint \mathrm{d} x \mathrm{~d} y W(x-y) s(x) s(y)+\int \mathrm{d} x \int_{0}^{s(x)} \mathrm{d} \rho \phi^{-1}[\rho]-A \int \mathrm{d} x s(x)
$$

The nonlinearity $\phi$ must be invertible in the range $(0, s)$ for any possible firing rate $s$. For $L$ to be bounded ${ }_{404}$ from below for a network of any size $N$, we need

1. $W(x)$ to be negative definite, and

2. $\int_{0}^{s} \mathrm{~d} \rho \phi^{-1}[\rho]-A s$ to be bounded from below for any possible firing rate $s$.

We can check that these hold for our particular functions. Equation 37 immediately shows that the first condition is met. Equation 34 states that $\phi^{-1}[\rho]=\rho$ when $\rho>0$, so $\int_{0}^{s} \mathrm{~d} \rho \phi^{-1}[\rho]-A s=\frac{1}{2} s^{2}-A s$, which satisfies the second condition.

Now we take the time derivative and use Eq. 39:

$$
\begin{aligned}
\frac{\mathrm{d} L}{\mathrm{~d} t} & =-\int \mathrm{d} x\left\{2 \int \mathrm{d} y W(x-y) s(y)-\phi^{-1}[s(x)]+A\right\} \frac{\mathrm{d} s(x)}{\mathrm{d} t} \\
& =-\tau \int \mathrm{d} x \frac{\mathrm{d} g(x)}{\mathrm{d} t} \frac{\mathrm{d} s(x)}{\mathrm{d} t} \\
& =-\tau \int \mathrm{d} x \phi^{\prime}[g(x)]\left(\frac{\mathrm{d} g(x)}{\mathrm{d} t}\right)^{2}
\end{aligned}
$$

As long as $\phi$ is a monotonically nondecreasing function, $\mathrm{d} L / \mathrm{d} t \leq 0$. Thus, $L$ is a Lyapunov functional.

Now we seek to simplify Eq. 60 . Suppose we are very close to a steady-state solution, so $\mathrm{d} g / \mathrm{d} t \approx 0$. We substitute Eq. 39 into Eq. 60 to obtain

$$
\begin{aligned}
L & =-\frac{1}{2} \int \mathrm{d} x[g(x)-A] s(x)+\int \mathrm{d} x \int_{0}^{s(x)} \mathrm{d} \rho \phi^{-1}[\rho]-A \int \mathrm{d} x s(x) \\
& =-\frac{1}{2} \int \mathrm{d} x g(x) s(x)+\int \mathrm{d} x \int_{0}^{s(x)} \mathrm{d} \rho \phi^{-1}[\rho]-\frac{A}{2} \int \mathrm{d} x s(x) .
\end{aligned}
$$

Now we invoke our ReLU $\phi$ from Eq. 34 to obtain

$$
L=-\frac{1}{2} \int \mathrm{d} x[g(x)-s(x)] s(x)-\frac{A}{2} \int \mathrm{d} x s(x)=-\frac{A}{2} \int \mathrm{d} x s(x)
$$


The last equality was obtained by noticing that for any $x$, either $s(x)=0$ or $g(x)-s(x)=0$ with our $\phi$. Therefore, the stable solution that minimizes $L$ is the one that maximizes the total firing rate.

We can apply our sinusoidal $g$ in Eq. 57 to perform the integral:

$$
L=-\frac{N A G}{2 \pi^{2}}(\sin \kappa z-\kappa z \cos \kappa z),
$$

where $N$ is the network size. So $L$ depends on $G$ and the quantity $\kappa z$, which we will rewrite as $\psi$. We now simplify Eq. 64 using Eq. 56:

$$
L=-\frac{N A^{2}(\sin \psi-\psi \cos \psi)}{4 \tilde{W}(0)(\sin \psi-\psi \cos \psi)-2 \pi \cos \psi}=-\frac{N A^{2}}{4 \tilde{W}(0)+\frac{2 \pi}{\psi-\tan \psi}} .
$$

Note that $1 /(\tan \psi-\psi)$ is a monotonically increasing function of $\psi$ in the range $[0, \pi]$, so to minimize $L$, we need to minimize $\psi$. Meanwhile, Eq. 56 now reads $\psi-\cos \psi \sin \psi=\pi / 2 \tilde{W}(\kappa)$. The left-hand side is also a monotonically increasing function of $\psi$ in the range $[0, \pi]$, so to minimize $\psi$, we need to maximize $\tilde{W}(\kappa)$. Thus, the Lyapunov stable wavelength $\lambda=2 \pi / \kappa$ is the one that maximizes $\tilde{W}(\kappa)$. This is the same mode that grows the fastest for the linearized dynamics in Eq. 44.

\section{Bump motion under drive and noise}

\section{Dynamics along the attractor manifold}

Now that we have determined the baseline configuration $g$, including the bump shape and bump distance, we investigate its motion under drive $b$ and noise $\zeta$. We introduce $\theta$ to label the position of the configuration. It can be defined as the center of mass or the point of maximum activity of one of the bumps. We expand the full time-dependent configuration with respect to the baseline configuration located at $\theta$ :

$$
g_{\alpha}(x, t)=g(x-\theta)+\delta g_{\alpha}(x, t)
$$

$g(x-\theta)$ solves Eq. 39 with $\mathrm{d} g / \mathrm{d} t=0$; to facilitate calculations below, we will write the baseline equation in 429 this form:

$$
g(x-\theta)=\sum_{\beta} \int \mathrm{d} y W_{\beta}(x, y) \phi[g(y-\theta)]+A .
$$

Substituting Eq. 66 into Eq. 32 and invoking Eq. 67, we obtain the following linearized dynamics for $\delta g$ :

$$
\tau \frac{\mathrm{d} \delta g_{\alpha}(x, t)}{\mathrm{d} t}+\delta g_{\alpha}(x, t)=\sum_{\beta} \int \mathrm{d} y W_{\beta}(x, y) \phi^{\prime}[g(y-\theta)] \delta g_{\beta}(y, t) \pm_{\alpha} \gamma b(t)+\zeta_{\alpha}(x, t) .
$$

We can rewrite this as

$$
\tau \frac{\mathrm{d} \delta g_{\alpha}(x, t)}{\mathrm{d} t}=\sum_{\beta} \int \mathrm{d} y K_{\alpha \beta}(x, y ; \theta) \delta g_{\beta}(y, t) \pm_{\alpha} \gamma b(t)+\zeta_{\alpha}(x, t),
$$

where

$$
K_{\alpha \beta}(x, y ; \theta)=W_{\beta}(x, y) \phi^{\prime}[g(y-\theta)]-\delta_{\alpha \beta} \delta(x-y)
$$

We will often suppress the argument of derivatives of $g$. If we consider a configuration located at $\theta, \mathrm{d} g / \mathrm{d} x \quad 434$ implies $\mathrm{d} g(x-\theta) / \mathrm{d} x$. We make the argument explicit when necessary. 
If we differentiate Eq. 67 by $\theta$, we obtain

$$
\begin{aligned}
\frac{\mathrm{d} g}{\mathrm{~d} x} & =\sum_{\beta} \int \mathrm{d} y W_{\beta}(x, y) \phi^{\prime}[g(y-\theta)] \frac{\mathrm{d} g}{\mathrm{~d} y} \\
0 & =\sum_{\beta} \int \mathrm{d} y K_{\alpha \beta}(x, y ; \theta) \frac{\mathrm{d} g}{\mathrm{~d} y}
\end{aligned}
$$

which indicates that $\mathrm{d} g / \mathrm{d} x$ is a right eigenvector of $K$ with eigenvalue 0 . To be explicit about this, we ${ }_{436}$ recover the discrete case by converting continuous functions to vectors and matrices:

$$
g_{i}=g(i-\theta), \quad \Delta g_{i}=\left.\frac{\mathrm{d} g(x-\theta)}{\mathrm{d} x}\right|_{x=i}, \quad K_{\alpha \beta i j}=K_{\alpha \beta}(i, j ; \theta) .
$$

If we concatenate matrices and vectors across populations as

$$
\mathrm{J}=\left(\begin{array}{ll}
\mathrm{K}_{\mathrm{LL}} & \mathrm{K}_{\mathrm{LR}} \\
\mathrm{K}_{\mathrm{RL}} & \mathrm{K}_{\mathrm{RR}}
\end{array}\right), \quad \mathbf{e}=\left(\begin{array}{c}
\Delta \mathrm{g} \\
\Delta \mathrm{g}
\end{array}\right)
$$

e is the right null eigenvector of $\mathrm{J}: 0=\sum_{j} J_{i j} e_{j}$.

Since $K$ is not symmetric, its left and right eigenvectors may be different. To find the left null eigenvector, we again differentiate Eq. 67 with respect to $\theta$, but this time interchanging variables $x$ and $y$ :

$$
\begin{aligned}
\frac{\mathrm{d} g}{\mathrm{~d} y} & =\sum_{\beta} \int \mathrm{d} x W_{\beta}(y, x) \phi^{\prime}[g(x-\theta)] \frac{\mathrm{d} g}{\mathrm{~d} x} \\
& \approx 2 \int \mathrm{d} x W(x-y) \phi^{\prime}[g(x-\theta)] \frac{\mathrm{d} g}{\mathrm{~d} x}
\end{aligned}
$$

The second equality is obtained from Eqs. 35 and 36. Replacing the position $y$ by $y \pm_{\beta} \xi$, where $\xi$ is the ${ }_{440}$ connectivity shift, we get

$$
\frac{\mathrm{d} g\left(y-\theta \pm_{\beta} \xi\right)}{\mathrm{d} y} \approx 2 \int \mathrm{d} x W(x-y \mp \beta \xi) \phi^{\prime}[g(x-\theta)] \frac{\mathrm{d} g(x-\theta)}{\mathrm{d} x},
$$

where we have made the arguments of $g$ explicit. Let's define shifted versions of the baseline $g$ for each ${ }_{442}$ population $\alpha$ :

$$
\bar{g}_{\alpha}(x)=g\left(x \pm_{\alpha} \xi\right)
$$

Since $\xi$ is small,

$$
\sum_{\alpha} \bar{g}_{\alpha}(x) \approx 2 g(x)
$$

Applying these expressions to Eq. 75 and recalling Eq. 35,

$$
\begin{aligned}
\frac{\mathrm{d} \bar{g}_{\beta}}{\mathrm{d} y} & \approx 2 \int \mathrm{d} x W_{\beta}(x, y) \phi^{\prime}[g(x-\theta)] \frac{\mathrm{d} g}{\mathrm{~d} x} \\
& \approx \sum_{\alpha} \int \mathrm{d} x W_{\beta}(x, y) \phi^{\prime}[g(x-\theta)] \frac{\mathrm{d} \bar{g}_{\alpha}}{\mathrm{d} x} .
\end{aligned}
$$


Finally, we multiply both sides of the equation by $\phi^{\prime}[g(y-\theta)]$ to obtain

$$
\begin{aligned}
\phi^{\prime}[g(y-\theta)] \frac{\mathrm{d} \bar{g}_{\beta}}{\mathrm{d} y} & \approx \sum_{\alpha} \int \mathrm{d} x W_{\beta}(x, y) \phi^{\prime}[g(y-\theta)] \phi^{\prime}[g(x-\theta)] \frac{\mathrm{d} \bar{g}_{\alpha}}{\mathrm{d} x} \\
0 & =\sum_{\alpha} \int \mathrm{d} x K_{\alpha \beta}(x, y ; \theta) \phi^{\prime}[g(x-\theta)] \frac{\mathrm{d} \bar{g}_{\alpha}}{\mathrm{d} x} .
\end{aligned}
$$

Thus $\phi^{\prime}[g(x-\theta)] \mathrm{d} \bar{g}_{\alpha} / \mathrm{d} x$ is the left null eigenvector for $K_{\alpha \beta}$. Again, to be explicit, the discrete equivalent is ${ }_{445}$

$$
\mathrm{J}=\left(\begin{array}{cc}
\mathrm{K}_{\mathrm{LL}} & \mathrm{K}_{\mathrm{LR}} \\
\mathrm{K}_{\mathrm{RL}} & \mathrm{K}_{\mathrm{RR}}
\end{array}\right), \quad \mathbf{f}=\left(\begin{array}{c}
\phi^{\prime}[\mathbf{g}] \odot \Delta \overline{\mathbf{g}}_{\mathrm{L}} \\
\phi^{\prime}[\mathbf{g}] \odot \Delta \overline{\mathbf{g}}_{\mathrm{R}}
\end{array}\right)
$$

where $\odot$ represents element-wise (Hadamard) multiplication. Then, $\mathbf{f}$ is the left null eigenvector of $\mathbf{J}$ : $0=\sum_{i} J_{i j} f_{i}$.

We now revisit Eq. 66 and assume that $g$ changes such that the bumps slowly move along the attractor manifold:

$$
\begin{gathered}
g_{\alpha}(x, t) \approx g(x-\theta(t)), \\
\frac{\mathrm{d} \delta g_{\alpha}(x, t)}{\mathrm{d} t}=\frac{\mathrm{d} g_{\alpha}(x, t)}{\mathrm{d} t} \approx-\frac{\mathrm{d} g(x-\theta(t))}{\mathrm{d} x} \frac{\mathrm{d} \theta}{\mathrm{d} t} .
\end{gathered}
$$

Again for simplicity, we will often suppress arguments of derivatives of $g$ and dependence on $t$. We return ${ }_{448}$ to Eq. 69 , project it along the left null eigenvector, and apply Eq. 81 to obtain

$$
-\tau \frac{\mathrm{d} \theta}{\mathrm{d} t} \sum_{\alpha} \int \mathrm{d} x \phi^{\prime}[g(x-\theta)] \frac{\mathrm{d} \bar{g}_{\alpha}}{\mathrm{d} x} \frac{\mathrm{d} g}{\mathrm{~d} x}=\gamma b \sum_{\alpha} \int \mathrm{d} x\left( \pm_{\alpha} 1\right) \cdot \phi^{\prime}[g(x-\theta)] \frac{\mathrm{d} \bar{g}_{\alpha}}{\mathrm{d} x}+\sum_{\alpha} \int \mathrm{d} x \phi^{\prime}[g(x-\theta)] \frac{\mathrm{d} \bar{g}_{\alpha}}{\mathrm{d} x} \zeta_{\alpha}(x) .
$$

The velocity of bump motion is given by $\mathrm{d} \theta / \mathrm{d} t$. It is

$$
\frac{\mathrm{d} \theta}{\mathrm{d} t} \approx-\frac{\gamma b \sum_{\alpha} \int \mathrm{d} x\left( \pm_{\alpha} 1\right) \cdot \phi^{\prime}[g(x-\theta)] \frac{\mathrm{d} \bar{g}_{\alpha}(x-\theta)}{\mathrm{d} x}}{2 \tau \int \mathrm{d} x \phi^{\prime}[g(x-\theta)]\left(\frac{\mathrm{d} g(x-\theta)}{\mathrm{d} x}\right)^{2}}-\frac{\sum_{\alpha} \int \mathrm{d} x \phi^{\prime}[g(x-\theta)] \frac{\mathrm{d} \bar{g}_{\alpha}(x-\theta)}{\mathrm{d} x} \zeta_{\alpha}(x)}{2 \tau \int \mathrm{d} x \phi^{\prime}[g(x-\theta)]\left(\frac{\mathrm{d} g(x-\theta)}{\mathrm{d} x}\right)^{2}},
$$

where we have made the arguments of $g$ explicit. This equation encapsulates all aspects of bump motion for our theoretical model. It includes dependence on both drive $b$ and noise $\zeta$, the latter of which is kept in a general form. We will proceed by considering specific cases of this equation.

\section{Path integration velocity $v_{\text {drive }}$ due to driving input $b$}

The noiseless case of Eq. 83 with $\zeta_{\alpha}(x)=0$ yields the bump velocity due to drive $b$, which is responsible for path integration:

$$
v_{\text {drive }}=-\frac{\gamma b \int \mathrm{d} x \phi^{\prime}[g(x-\theta)]\left(\frac{\mathrm{d} \bar{g}_{\mathrm{R}}}{\mathrm{d} x}-\frac{\mathrm{d} \bar{g}_{\mathrm{L}}}{\mathrm{d} x}\right)}{2 \tau \int \mathrm{d} x \phi^{\prime}[g(x-\theta)]\left(\frac{\mathrm{d} g}{\mathrm{~d} x}\right)^{2}} .
$$


Note that this expression is independent of the position $\theta$. We can explicitly remove $\theta$ by shifting the dummy variable $x \rightarrow x+\theta$ :

$$
\begin{aligned}
v_{\text {drive }} & =-\frac{\gamma b \int \mathrm{d} x \phi^{\prime}[g(x)]\left(\frac{\mathrm{d} g(x+\xi)}{\mathrm{d} x}-\frac{\mathrm{d} g(x-\xi)}{\mathrm{d} x}\right)}{2 \tau \int \mathrm{d} x \phi^{\prime}[g(x)]\left(\frac{\mathrm{d} g(x)}{\mathrm{d} x}\right)^{2}} \\
\approx & -\frac{\gamma b \xi \int \mathrm{d} x \phi^{\prime}[g(x)] \frac{\mathrm{d}^{2} g}{\mathrm{~d} x^{2}}}{\tau \int \mathrm{d} x \phi^{\prime}[g(x)]\left(\frac{\mathrm{d} g}{\mathrm{~d} x}\right)^{2}} .
\end{aligned}
$$

Now let's consider the specific ReLU activation function $\phi$. Equation 34 implies

$$
\phi^{\prime}[g]=\left\{\begin{array}{ll}
0 & g \leq 0 \\
1 & g>0,
\end{array} \quad \text { so } \quad \phi^{\prime}[g]^{2}=\phi^{\prime}[g] \quad \text { and } \quad \phi^{\prime}[g] \cdot \phi[g]=\phi[g] .\right.
$$

These identities, along with the definition for $s$ (Eq. 33), give

$$
\phi^{\prime}[g(x)] \frac{\mathrm{d}^{2} g}{\mathrm{~d} x^{2}}=\frac{\mathrm{d}^{2} s}{\mathrm{~d} x^{2}}, \quad \phi^{\prime}[g(x)]\left(\frac{\mathrm{d} g}{\mathrm{~d} x}\right)^{2}=\left(\frac{\mathrm{d} s}{\mathrm{~d} x}\right)^{2}, \quad \phi[g(x)]\left(\frac{\mathrm{d} g}{\mathrm{~d} x}\right)^{2}=s(x)\left(\frac{\mathrm{d} s}{\mathrm{~d} x}\right)^{2} .
$$

Applying the first two equalities to Eq. 85 produces Eq. 8 of the Results section.

Now we reintroduce noise $\zeta$ and assume it is independent across neurons and timesteps, with mean $\langle\zeta\rangle$. If we average Eq. 83 over $\zeta$, the numerator of the second term becomes

$$
\sum_{\alpha} \int \mathrm{d} x \phi^{\prime}[g(x-\theta)] \frac{\mathrm{d} \bar{g}_{\alpha}(x-\theta)}{\mathrm{d} x}\langle\zeta\rangle=0 .
$$

The integral vanishes because $g$ is even and $\sum_{\alpha} \mathrm{d} \bar{g}_{\alpha} / \mathrm{d} x$ is odd. Thus,

$$
\left\langle\frac{\mathrm{d} \theta}{\mathrm{d} t}\right\rangle=v_{\text {drive }}
$$

demonstrating that networks with independent noise still path integrate on average.

\section{Diffusion $D_{\text {input }}$ due to input noise}

Independent noise $\zeta$ produces diffusion, a type of deviation in bump motion away from the average trajectory. It is quantified by the diffusion coefficient $D$ :

$$
\left\langle[\theta(t)-\langle\theta(t)\rangle]^{2}\right\rangle=2 D t
$$

In terms of derivatives of $\theta$,

$$
\left\langle[\theta(t)-\langle\theta(t)\rangle]^{2}\right\rangle=\int_{0}^{t} \int_{0}^{t} \mathrm{~d} t^{\prime} \mathrm{d} t^{\prime \prime}\left\langle\left(\frac{\mathrm{d} \theta}{\mathrm{d} t^{\prime}}-\left\langle\frac{\mathrm{d} \theta}{\mathrm{d} t^{\prime}}\right\rangle\right)\left(\frac{\mathrm{d} \theta}{\mathrm{d} t^{\prime \prime}}-\left\langle\frac{\mathrm{d} \theta}{\mathrm{d} t^{\prime \prime}}\right\rangle\right)\right\rangle .
$$


Equations 83 and 89 imply

$$
\frac{\mathrm{d} \theta}{\mathrm{d} t}-\left\langle\frac{\mathrm{d} \theta}{\mathrm{d} t}\right\rangle=-\frac{\sum_{\alpha} \int \mathrm{d} x \phi^{\prime}[g(x-\theta)] \frac{\mathrm{d} \bar{g}_{\alpha}}{\mathrm{d} x} \zeta_{\alpha}(x)}{2 \tau \int \mathrm{d} x \phi^{\prime}[g(x-\theta)]\left(\frac{\mathrm{d} g}{\mathrm{~d} x}\right)^{2}} .
$$

We then shift the dummy variable $x \rightarrow x+\theta(t)$ and reintroduce explicit dependence on $t$ to obtain

$$
\left\langle[\theta(t)-\langle\theta(t)\rangle]^{2}\right\rangle=\int_{0}^{t} \int_{0}^{t} \mathrm{~d} t^{\prime} \mathrm{d} t^{\prime \prime} \frac{\sum_{\alpha \beta} \iint \mathrm{d} x \mathrm{~d} y \phi^{\prime}[g(x)] \phi^{\prime}[g(y)] \frac{\mathrm{d} \bar{g}_{\alpha}}{\mathrm{d} x} \frac{\mathrm{d} \bar{g}_{\beta}}{\mathrm{d} y}\left\langle\zeta_{\alpha}\left(x+\theta\left(t^{\prime}\right), t^{\prime}\right) \zeta_{\beta}\left(y+\theta\left(t^{\prime \prime}\right), t^{\prime \prime}\right)\right\rangle}{4 \tau^{2}\left[\int \mathrm{d} x \phi^{\prime}[g(x)]\left(\frac{\mathrm{d} g}{\mathrm{~d} x}\right)^{2}\right]^{2}} .
$$

One class of independent $\zeta$ is Gaussian noise added to the total synaptic input, which represents neural fluctuations at short timescales. We assume it is independent across neurons and timesteps with zero mean and fixed variance $\sigma^{2}$ :

$$
\left\langle\zeta_{\alpha}(x, t)\right\rangle=0, \quad\left\langle\zeta_{\alpha}(x, t) \zeta_{\beta}\left(y, t^{\prime}\right)\right\rangle=\sigma^{2} \Delta t \delta\left(t-t^{\prime}\right) \delta_{\alpha \beta} \delta(x-y) .
$$

$\Delta t$ is the simulation timestep, which defines the rate at which the random noise variable is resampled. Equation 93 then becomes, with the help of Eq. 77,

$$
\begin{aligned}
\left\langle[\theta(t)-\langle\theta(t)\rangle]^{2}\right\rangle & =\int_{0}^{t} \mathrm{~d} t^{\prime} \frac{\sigma^{2} \Delta t \sum_{\alpha} \int \mathrm{d} x \phi^{\prime}[g(x)]^{2}\left(\frac{\mathrm{d} \bar{g}_{\alpha}}{\mathrm{d} x}\right)^{2}}{4 \tau^{2}\left[\int \mathrm{d} x \phi^{\prime}[g(x)]\left(\frac{\mathrm{d} g}{\mathrm{~d} x}\right)^{2}\right]^{2}} \\
& \approx \frac{\sigma^{2} \Delta t \int \mathrm{d} x \phi^{\prime}[g(x)]^{2}\left(\frac{\mathrm{d} g}{\mathrm{~d} x}\right)^{2}}{2 \tau^{2}\left[\int \mathrm{d} x \phi^{\prime}[g(x)]\left(\frac{\mathrm{d} g}{\mathrm{~d} x}\right)^{2}\right]^{2}} \cdot t
\end{aligned}
$$

Reconciling this with the definition of the diffusion coefficient $D$ in Eq. 90 yields

$$
D_{\text {input }}=\frac{\sigma^{2} \Delta t \int \mathrm{d} x \phi^{\prime}[g(x)]^{2}\left(\frac{\mathrm{d} g}{\mathrm{~d} x}\right)^{2}}{4 \tau^{2}\left[\int \mathrm{d} x \phi^{\prime}[g(x)]\left(\frac{\mathrm{d} g}{\mathrm{~d} x}\right)^{2}\right]^{2}}
$$

Applying Eq. 87 for a ReLU $\phi$ gives Eq. 10 of the Results section.

\section{Diffusion $D_{\text {spike }}$ due to spiking noise}

Instead of input noise, we consider independent noise arising from spiking neurons. In this case, the stochastic firing rate $s$ is no longer the deterministic expression in Eq. 33. Instead,

$$
s_{\alpha}(x, t)=\frac{c_{\alpha}(x, t)}{\Delta t}
$$


where $c$ is the number of spikes emitted in a simulation timestep of length $\Delta t$. We model each $c_{\alpha}(x, t)$ as an independent Poisson-like random variable driven by the deterministic firing rate $\phi\left[g_{\alpha}(x, t)\right]$ with Fano factor $F$. It has mean $\phi\left[g_{\alpha}(x, t)\right] \Delta t$ and variance $F \phi\left[g_{\alpha}(x, t)\right] \Delta t$. Therefore,

$$
s_{\alpha}(x, t)=\phi\left[g_{\alpha}(x, t)\right]+\sqrt{\frac{F \phi\left[g_{\alpha}(x, t)\right]}{\Delta t}} \eta_{\alpha}(x, t),
$$

where each $\eta_{\alpha}(x, t)$ is an independent random variable with zero mean and unit variance:

$$
\left\langle\eta_{\alpha}(x, t)\right\rangle=0, \quad\left\langle\eta_{\alpha}(x, t) \eta_{\beta}\left(y, t^{\prime}\right)\right\rangle=\Delta t \delta\left(t-t^{\prime}\right) \delta_{\alpha \beta} \delta(x-y) .
$$

As in Eq. 94, the simulation timestep $\Delta t$ defines the rate at which $\eta$ is resampled. By substituting Eq. 98 into Eq. 32, we see that spiking neurons can be described by deterministic firing rate dynamics with the stochastic noise term

$$
\zeta_{\alpha}(x, t)=\sum_{\beta} \int \mathrm{d} y W_{\beta}(x, y) \sqrt{\frac{F \phi\left[g_{\beta}(y, t)\right]}{\Delta t}} \eta_{\beta}(y, t) .
$$

Now we calculate the diffusion coefficient produced by this noise. Equation 92 becomes

$$
\begin{aligned}
\frac{\mathrm{d} \theta}{\mathrm{d} t}-\left\langle\frac{\mathrm{d} \theta}{\mathrm{d} t}\right\rangle & =-\frac{\sum_{\alpha \beta} \iint \mathrm{d} x \mathrm{~d} y W_{\beta}(x, y) \phi^{\prime}[g(x-\theta)] \frac{\mathrm{d} \bar{g}_{\alpha}}{\mathrm{d} x} \sqrt{\frac{F \phi[g(y-\theta)]}{\Delta t}} \eta_{\beta}(y)}{2 \tau \int \mathrm{d} x \phi^{\prime}[g(x-\theta)]\left(\frac{\mathrm{d} g}{\mathrm{~d} x}\right)^{2}} \\
& =-\frac{\sum_{\beta} \int \mathrm{d} y \frac{\mathrm{d} \bar{g}_{\beta}}{\mathrm{d} y} \sqrt{\frac{F \phi[g(y-\theta)]}{\Delta t}} \eta_{\beta}(y)}{2 \tau \int \mathrm{d} x \phi^{\prime}[g(x-\theta)]\left(\frac{\mathrm{d} g}{\mathrm{~d} x}\right)^{2}}
\end{aligned}
$$

We used Eq. 78 to obtain the second equality. We then proceed as for input noise to calculate

$$
\left\langle[\theta(t)-\langle\theta(t)\rangle]^{2}\right\rangle=\int_{0}^{t} \int_{0}^{t} \mathrm{~d} t^{\prime} \mathrm{d} t^{\prime \prime} \frac{\frac{F}{\Delta t} \sum_{\alpha \beta} \iint \mathrm{d} x \mathrm{~d} y \sqrt{\phi[g(x)] \phi[g(y)]} \frac{\mathrm{d} \bar{g}_{\alpha}}{\mathrm{d} x} \frac{\mathrm{d} \bar{g}_{\beta}}{\mathrm{d} y}\left\langle\eta_{\alpha}\left(x+\theta\left(t^{\prime}\right), t^{\prime}\right) \eta_{\beta}\left(y+\theta\left(t^{\prime \prime}\right), t^{\prime \prime}\right)\right\rangle}{4 \tau^{2}\left[\int \mathrm{d} x \phi^{\prime}[g(x)]\left(\frac{\mathrm{d} g}{\mathrm{~d} x}\right)^{2}\right]^{2}},
$$

which yields the diffusion coefficient

$$
D_{\text {spike }}=\frac{F \int \mathrm{d} x \phi[g(x)]\left(\frac{\mathrm{d} g}{\mathrm{~d} x}\right)^{2}}{4 \tau^{2}\left[\int \mathrm{d} x \phi^{\prime}[g(x)]\left(\frac{\mathrm{d} g}{\mathrm{~d} x}\right)^{2}\right]^{2}}
$$

After applying Eq. 87 for a ReLU $\phi$ and setting $F=1$ for Poisson spiking, we obtain Eq. 20 of the Results 485 section. 


\section{Drift velocity $v_{\text {conn }}(\theta)$ due to quenched connectivity noise}

Suppose that we perturb the symmetric, translation-invariant $W$ by a small component $V$ representing deviations away from an ideal attractor architecture:

$$
W_{\beta}(x, y) \rightarrow W_{\beta}(x, y)+V_{\alpha \beta}(x, y) .
$$

By Eq. 32, this produces the noise term

$$
\zeta_{\alpha}(x, t)=\sum_{\beta} \int \mathrm{d} y V_{\alpha \beta}(x, y) \phi\left[g_{\beta}(y, t)\right] .
$$

In contrast to input and spiking noise, this noise is correlated across neurons and time, so it cannot be averaged away as in Eqs. 88 and 89. Substituting Eq. 105 into Eq. 83, we obtain

$$
\frac{\mathrm{d} \theta}{\mathrm{d} t}=v_{\text {drive }}+v_{\text {conn }}(\theta)
$$

where the drift velocity is

$$
v_{\text {conn }}(\theta)=-\frac{\sum_{\alpha \beta} \iint \mathrm{d} x \mathrm{~d} y V_{\alpha \beta}(x, y) \phi^{\prime}[g(x-\theta)] \frac{\mathrm{d} g(x-\theta)}{\mathrm{d} x} \phi[g(y-\theta)]}{2 \tau \int \mathrm{d} x \phi^{\prime}[g(x-\theta)]\left(\frac{\mathrm{d} g(x-\theta)}{\mathrm{d} x}\right)^{2}} .
$$

We have made the dependence on bump position $\theta$ explicit to illustrate how it influences $v_{\text {conn }}(\theta)$. After applying Eq. 87 for a ReLU $\phi$, we obtain Eq. 23 of the Results section.

We now make scaling arguments for speed difference (Eq. 29), speed variability (Eq. 30), and escape drive $b_{0}$ (Eq. 25). To do so, we impose a ReLU $\phi$ and return to discrete variables to be explicit:

$$
v_{\mathrm{conn} ; \theta}=-\frac{\sum_{\alpha \beta} \sum_{i j} V_{\alpha \beta i j} \cdot \Delta s_{i-\theta} \cdot s_{j-\theta}}{2 \tau \sum_{i}\left(\Delta s_{i-\theta}\right)^{2}} .
$$

We need to understand how the numerator scales with $M$ and $N$. It is a weighted sum of $4 N^{2}$ independent Gaussian random variables $V_{\alpha \beta i j}$ and is thus a Gaussian random variable itself. It has zero mean, but its variance is proportional to $N^{2} \cdot M^{2} / N^{2}$. The $N^{2}$ comes from the number of terms in the sum and the $M^{2} / N^{2}$ comes from the scaling of $\mathrm{d} s / \mathrm{d} x$ (Eq. 11). In combination with the scaling of the denominator, we conclude that $v_{\mathrm{conn} ; \theta}$ is a Gaussian random variable with

$$
\mathrm{E}\left[v_{\text {conn } ; \theta}\right]=0, \quad \operatorname{Var}\left[v_{\text {conn } ; \theta}\right] \propto \frac{N^{2}}{M^{2}} .
$$

Equation 108 implies that $v_{\text {conn; } \theta}$ is correlated over $\theta$. The weights for the sum over $V_{\alpha \beta i j}$ are the firing rates and their derivatives for a bump centered at $\theta$. If $\theta$ is slightly changed, almost the same entries of $V$ will be summed over with similar weights. The amount of correlation across $\theta$ is determined by the degree of overlap in weights, and therefore, by the width and number of bumps. Let's consider the effects of changing $N$ and $M$ on the covariance matrix $\operatorname{Cov}\left[v_{\operatorname{conn} ; \theta}, v_{\operatorname{conn} ; \theta^{\prime}}\right]$. A larger $N$ increases the bump width and the correlation length proportionally, so values of the main diagonal decay proportionally more slowly into the off diagonals. A larger $M$ redistributes values among the diagonals by decreasing the bump width and adding 
more bumps, but it does not change the total amount of correlation. Thus,

$$
\sum_{\theta, \theta^{\prime}} \operatorname{Cov}\left[v_{\operatorname{conn} ; \theta}, v_{\text {conn } ; \theta^{\prime}}\right] \propto N^{2} \cdot \operatorname{Var}\left[v_{\operatorname{conn} ; \theta}\right]
$$

This allows us to evaluate

$$
\operatorname{Var}\left[\underset{\theta}{\operatorname{mean}} v_{\text {conn } ; \theta}\right]=\operatorname{Var}\left[\frac{1}{N} \sum_{\theta} v_{\text {conn } ; \theta}\right]=\frac{1}{N^{2}} \sum_{\theta, \theta^{\prime}} \operatorname{Cov}\left[v_{\text {conn } ; \theta}, v_{\text {conn } ; \theta^{\prime}}\right] \propto \frac{N^{2}}{M^{2}}
$$

As a sum of zero-mean Gaussian random variables, $\operatorname{mean}_{\theta} v_{\text {conn; } \theta}$ is also a zero-mean Gaussian random 512 variable. That means $\left|\operatorname{mean}_{\theta} v_{\operatorname{conn} ; \theta}\right|$ follows a folded normal distribution, which obeys

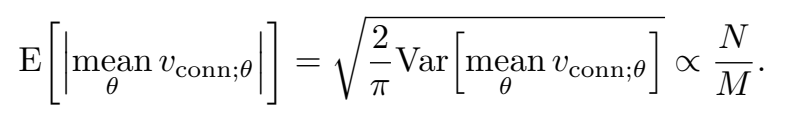

Combining this with Eqs. 12 and 14 produces the scalings for speed difference in Eq. 31.

We now study speed variability, which involves the expression

$$
\operatorname{std} v_{\operatorname{conn} ; \theta}=\sqrt{\frac{1}{N} \sum_{\theta} v_{\mathrm{conn} ; \theta}^{2}}
$$

Since each $v_{\text {conn; } \theta}$ is Gaussian, the sum of their squares follows a generalized chi-square distribution. Its 516 mean is the trace of the covariance matrix $\operatorname{Cov}\left[v_{\operatorname{conn} ; \theta}, v_{\operatorname{conn} ; \theta^{\prime}}\right]$, which is equal to $N$ times the variance. 517 Thus, by Eq. 109,

$$
\mathrm{E}\left[\frac{1}{N} \sum_{\theta} v_{\text {conn } ; \theta}^{2}\right]=\frac{1}{N} \cdot N \cdot \operatorname{Var}\left[v_{\text {conn } ; \theta}\right] \propto \frac{N^{2}}{M^{2}}
$$

We are interested in the square root of the random variable on the left-hand side, and we anticipate its 519 expected value to scale as the square root of the right-hand side. We can make this argument precise. 520 Suppose $H$ is a random variable with a probability distribution function $p(h)$ that scales with a power of 521 the parameter $B$. We can write

$$
p(h)=B^{n} P\left(B^{m} h\right)
$$

for exponents $n$ and $m$, where the rescaled probability distribution function $P$ does not scale with $B$. 523 Conservation of total probability implies

$$
B^{n} \int \mathrm{d} h P\left(B^{m} h\right)=B^{n} B^{-m} \int \mathrm{d} h^{\prime} P\left(h^{\prime}\right)=1 .
$$

Thus, $m=n$. Next, suppose we know that $\mathrm{E}[H] \propto B^{o}$ :

$$
\mathrm{E}[H]=B^{n} \int \mathrm{d} h h P\left(B^{n} h\right)=B^{-n} \int \mathrm{d} h^{\prime} h^{\prime} P\left(h^{\prime}\right) \propto B^{o} .
$$

Thus, $n=-o$. We can now conclude that $\mathrm{E}[\sqrt{H}] \propto \sqrt{\mathrm{E}[H]}$ :

$$
\mathrm{E}[\sqrt{H}]=B^{-o} \int \mathrm{d} h \sqrt{h} P\left(B^{-o} h\right)=B^{o / 2} \int \mathrm{d} h^{\prime} \sqrt{h^{\prime}} P\left(h^{\prime}\right) \propto B^{o / 2} .
$$


Applying this result to Eq. 114, we obtain

$$
\mathrm{E}\left[\underset{\theta}{\operatorname{std}} v_{\text {conn } ; \theta}\right]=\mathrm{E}\left[\sqrt{\left.\frac{1}{N} \sum_{\theta} v_{\operatorname{conn} ; \theta}^{2}\right]} \propto \sqrt{\mathrm{E}\left[\frac{1}{N} \sum_{\theta} v_{\operatorname{conn} ; \theta}^{2}\right]} \propto \frac{N}{M} .\right.
$$

Combining this with Eqs. 12 and 14 produces the scalings for speed variability in Eq. 31.

The escape drive $b_{0}$ involves the expression $\max _{\theta}\left|v_{\text {conn; } ; \theta}\right|$. Extreme value statistics for correlated random variables is generally poorly understood. We follow Majumdar et al. (2020) and provide a heuristic argument for its scaling. We can partition $v_{\text {conn; } \theta}$ across $\theta$ into groups that are largely independent from one another based on its correlation structure. As discussed above, $v_{\mathrm{conn} ; \theta}$ is a weighted sum of independent Gaussian random variables $V_{\alpha \beta i j}$ (Eq. 108). The weights are products between the firing rates $s_{j-\theta}$ and their derivatives $\Delta s_{i-\theta}$ for a configuration centered at position $\theta$. If we choose two $\theta$ 's such that bumps do not overlap, the

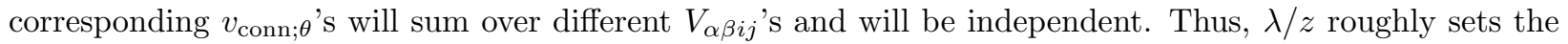
number of independent components, where $\lambda$ is the bump distance and $z$ is the bump width. This ratio does not change with $M$ or $N$ in our networks (Fig. $2 \mathrm{~F}$ ), so the maximum function does not change the scaling of $\left|v_{\operatorname{conn} ; \theta}\right|$ :

$$
\max _{\theta}\left|v_{\text {conn } ; \theta}\right| \propto\left|v_{\text {conn; } ; \theta}\right| \text {. }
$$

The scaling of $\mathrm{E}\left[\left|v_{\mathrm{conn} ; \theta}\right|\right]$ can be determined from $\operatorname{Var}\left[v_{\mathrm{conn} ; \theta}\right]$ through arguments similar to those made in ${ }_{539}$ Eqs. 115 to 118. Suppose we know that $\operatorname{Var}[H] \propto B^{o}$ and $\mathrm{E}[H]=0$. Then,

$$
\operatorname{Var}[H]=B^{n} \int \mathrm{d} h h^{2} P\left(B^{n} h\right)=B^{-2 n} \int \mathrm{d} h^{\prime}\left(h^{\prime}\right)^{2} P\left(h^{\prime}\right) \propto B^{o} .
$$

Thus, $n=-o / 2$. We can now conclude that $\mathrm{E}[|H|] \propto \sqrt{\operatorname{Var}[H]}$ :

$$
\mathrm{E}[|H|]=B^{-o / 2} \int \mathrm{d} h|h| P\left(B^{-o / 2} h\right)=B^{o / 2} \int \mathrm{d} h^{\prime}\left|h^{\prime}\right| P\left(h^{\prime}\right) \propto B^{o / 2} .
$$

Applying this result to Eq. 120, we obtain

$$
\mathrm{E}\left[\max _{\theta}\left|v_{\text {conn } ; \theta}\right|\right] \propto \mathrm{E}\left[\left|v_{\text {conn } ; \theta}\right|\right] \propto \sqrt{\operatorname{Var}\left[v_{\text {conn } ; \theta}\right]} \propto \frac{N}{M} .
$$

Combining this with Eqs. 12, 13 and 25 produces the scalings for the escape drive $b_{0}$ in Eq. 26.

\section{Simulation methods}

\section{Dynamics and parameter values}

To simulate the dynamics Eq. 32, we discretize the network by replacing neural position $x$ with index $i$ and propagate forward in time with the simple Euler method:

$$
\tau \frac{g_{\alpha i}(t+\Delta t)-g_{\alpha i}(x, t)}{\Delta t}+g_{\alpha i}(x, t)=\sum_{\beta j} W_{\beta i j} s_{\beta j}(t)+A \pm_{\alpha} \gamma b(t)+\zeta_{\alpha i}(t) .
$$

We use $\tau=10 \mathrm{~ms}$. We use $\Delta t=0.5 \mathrm{~ms}$ and $A=1$ for all simulations except those with spiking neurons. In the latter case, we use finer timesteps $\Delta t=0.1 \mathrm{~ms}$ and set $A=0.1 \mathrm{~ms}^{-1}$. Synaptic inputs $g$ and resting inputs $A$ can be dimensionless for rate-based simulations, but they must have units of rate for spiking simulations. We use $\gamma=0.1$ for rate-based simulations and $\gamma=0.01 \mathrm{~ms}^{-1}$ for spiking simulations. To achieve the relationship in Eq. 13 for circular mapping, we rescale $\gamma$ with network size $N$ and bump number 
$M$ :

$$
\gamma \rightarrow \gamma \cdot \frac{N}{600} \cdot \frac{3}{M}
$$

The connectivity $W$ takes the form in Eq. 37. Unless otherwise specified, we use shift $\xi=2$. To produce $M$ bumps in a network of size $N$, we turn to Eq. 46 and set $l=0.44 N / M$. We use $w=8 M / N \approx 3.5 / l$. For the case of $2 l>N / 2$, which corresponds to a one-bump network, the tails of the cosine function extend beyond the network size. Instead of truncating them, we wrap them around the ring:

$$
W(x) \rightarrow W(x)+W(x-N)+W(x+N) .
$$

This procedure, along with the scaling of $w$ with $N$ and $M$, accomplishes Eq. 7 and keeps the total connectivity strength per neuron $\sum_{i} W_{i}$ constant across all $N$ and $M$, where $W_{i}$ is the discrete form of $W(x)$.

To generate the Poisson-like spike counts $c_{\alpha i}(t)$ in Eq. 97, we rescale Poisson random variables:

$$
c_{\alpha i}(t)=F \cdot C_{\alpha i}(t), \quad C_{\alpha i}(t) \sim \operatorname{Pois}\left[\phi\left[g_{\alpha i}(t)\right] \Delta t / F\right] .
$$

These counts will be multiples of the Fano factor $F$. To produce a $c_{\alpha i}(t)$ whose domain is the natural numbers, one can follow Burak and Fiete (2009), who take multiple samples of $C_{\alpha i}(t)$ during each timestep.

To obtain theoretical values in Figs. 3 and 5 to 7 , we need to substitute the baseline inputs $g_{i}$ into the appropriate equations. We use noiseless and driveless simulations to generate $g_{i}$ instead of using Eq. 4 .

\section{Bump position}

We track the position $\theta$ of each bump using the firing rate summed across both populations $S_{i}(t)=$ $\sum_{\alpha} \phi\left[g_{\alpha i}(t)\right]$. We first estimate the positions of all the bumps by partitioning the network into segments of length $\lfloor N / M\rfloor$. If $N / M$ is not an integer, we skip one neuron between some segments to have them distributed as evenly as possible throughout the network. We sum $S_{i}(t)$ across all the segments and find the position $i_{0}$ with maximum value. We perform a circular shift of the original $S_{i}(t)$ such that $i_{0}$ is shifted to the middle of the first segment $\lfloor N / 2 M\rfloor$. The purpose of this process is to approximately center each bump within a segment so that $S_{i}(t)$ drops to 0 before reaching segment boundaries. We then calculate the center of mass of $S_{i}(t)$ within each segment. After reversing the circular shift, these centers of masses are taken to be the bump positions.

As an alternative, we can obtain a bump position between 0 and $N / M$ by simply computing the circular mean of $S_{i}(t)$ with periodicity $N / M$. However, this method does not track the position of each bump, so we do not use it.

\section{Path integration velocity and diffusion}

To obtain our results in Figs. 3 and 5 , we run each simulation for $T=5 \mathrm{~s}$. To extract the bump velocity $v$ produced by a constant drive $b$, we calculate the mean displacement $\Theta$ as a function of time offset $u$ :

$$
\Theta(u)=\frac{\Delta t}{T-u} \sum_{t}[\theta(t+u)-\theta(t)]
$$

$\theta$ is the bump position. This equation averages over fiducial starting times $t$, which ranges from 0 to $T-u-\Delta t$ in increments of $\Delta t$. We vary $u$ between 0 and $T / 2$ in increments of $\Delta t$; the maximum is $T / 2$ to ensure enough $t$ 's for accurate averaging. We then fit $\Theta(u)$ to a line through the origin to obtain the velocity:

$$
\Theta(u) \approx v u \text {. }
$$

We calculate the diffusion coefficient $D$ based on an ensemble of replicate simulations. In this section, ${ }_{584}$ angle brackets will indicate averaging over this ensemble. Following the definition of $D$ in Eq. 91, we calculate 585 
each bump's position relative to the mean motion of the ensemble:

$$
\omega(t)=\theta(t)-\langle\theta(t)\rangle
$$

We compute squared displacements and then average over fiducial starting times to obtain a mean squared displacement for each bump as a function of time offset $u$ :

$$
\Omega(u)=\frac{\Delta t}{T-u} \sum_{t}[\omega(t+u)-\omega(t)]^{2} .
$$

$t$ and $u$ span the same time ranges as they did for $\Theta$. We average $\Omega(u)$ over the ensemble and fit it to a line through the origin to obtain the diffusion coefficient:

$$
\langle\Omega(u)\rangle \approx 2 D u
$$

For simulations with $M$ bumps, we arbitrarily assign identity numbers $1, \ldots, M$ to bumps in each simulation. We perform ensemble averaging over bumps with the same identity numbers; that is, we only average over one bump per simulation. This way, we obtain separate values for each bump in Fig. 3E-H; nevertheless, these values lie on top of each other. In Fig. 3B, C, each point represents $v$ averaged across bumps. To calculate the mean velocity $\langle v\rangle$ in Fig. 3E, F, we fit $\langle\Theta(u)\rangle$ to a line through the origin. To estimate standard deviations for Fig. 3E-H and Fig. 5, we create 48 bootstrapped ensembles, each of which contains 48 replicate simulations sampled with replacement from the original ensemble. We calculate $\langle v\rangle$ or $D$ for each bootstrapped ensemble and record the resulting standard deviation. In Fig. 5, each point represents $D$ and its estimated standard deviation averaged across bumps.

\section{Trapping and position-dependent velocity}

For simulations with connectivity noise, we determine the escape drive $b_{0}$ (Fig. 6), the smallest drive that allows the bumps to travel through the entire network, by a binary search over $b$. We perform 8 rounds of search between the limits 0 and 1.28 and another 8 rounds between 0 and -1.28 to obtain $b_{0}$ within an accuracy of 0.01 . In each round, we run a simulation with the test $b$ and see whether the bumps travel through the network or get trapped. Traveling through the network means that every position (rounded to the nearest integer) has been visited by a bump, and trapping means that the motion of at least one bump slows below a threshold for a length of time.

To obtain the position-dependent bump velocity $v(\theta)$ produced by connectivity noise when $|b|>b_{0}$, we run a simulation until the bumps have traveled through the network. At each timestep, we record the positions of the bumps (binned to the nearest integer) and their instantaneous velocities with respect to the previous timestep. We smooth the velocities in time with a Gaussian kernel of width $10 \mathrm{~ms}$, which is the neural time constant $\tau$. We calculate the mean and standard deviation of these smoothed velocities for each position bin.

\section{Acknowledgments}

We are grateful to Steven Lee for sharing his code and to John Widloski for a careful reading of this manuscript.

\section{References}

S.-i. Amari. Dynamics of pattern formation in lateral-inhibition type neural fields. Biol. Cybern., 27(2): 77-87, 1977. 
R. Ben-Yishai, R. L. Bar-Or, and H. Sompolinsky. Theory of orientation tuning in visual cortex. Proc. Natl. Acad. Sci. U.S.A., 92(9):3844-3848, 1995.

C. D. Brody, R. Romo, and A. Kepecs. Basic mechanisms for graded persistent activity: discrete attractors, continuous attractors, and dynamic representations. Curr. Opin. Neurobiol., 13(2):204-211, 2003.

Y. Burak and I. R. Fiete. Accurate path integration in continuous attractor network models of grid cells. PLOS Comput. Biol., 5(2):e1000291, 2009.

Y. Burak and I. R. Fiete. Fundamental limits on persistent activity in networks of noisy neurons. Proc. Natl. Acad. Sci. U.S.A., 109(43):17645-17650, 2012.

D. Bush and N. Burgess. A hybrid oscillatory interference/continuous attractor network model of grid cell firing. J. Neurosci., 34(14):5065-5079, 2014.

S. C. Cannon, D. A. Robinson, and S. Shamma. A proposed neural network for the integrator of the oculomotor system. Biol. Cybern., 49(2):127-136, 1983.

A. Compte, N. Brunel, P. S. Goldman-Rakic, and X.-J. Wang. Synaptic mechanisms and network dynamics underlying spatial working memory in a cortical network model. Cereb. Cortex, 10(9):910-923, 2000.

C. Constantinidis and X.-J. Wang. A neural circuit basis for spatial working memory. Neuroscientist, 10(6): 553-565, 2004.

G. B. Ermentrout and J. D. Cowan. A mathematical theory of visual hallucination patterns. Biol. Cybern., 34(3):137-150, 1979.

I. R. Fiete, Y. Burak, and T. Brookings. What grid cells convey about rat location. J. Neurosci., 28(27): 6858-6871, 2008.

M. C. Fuhs and D. S. Touretzky. A spin glass model of path integration in rat medial entorhinal cortex. $J$. Neurosci., 26(16):4266-4276, 2006.

C. C. A. Fung, K. Y. M. Wong, and S. Wu. A moving bump in a continuous manifold: a comprehensive study of the tracking dynamics of continuous attractor neural networks. Neural Comput., 22(3):752-792, 2010.

R. J. Gardner, L. Lu, T. Wernle, M.-B. Moser, and E. I. Moser. Correlation structure of grid cells is preserved during sleep. Nat. Neurosci., 22(4):598-608, 2019.

R. J. Gardner, E. Hermansen, M. Pachitariu, Y. Burak, N. A. Baas, B. A. Dunn, M.-B. Moser, and E. I. Moser. Toroidal topology of population activity in grid cells. Nature, 602(7895):123-128, 2022.

J. Goldstone. Field theories with Superconductor solutions. Nuovo Cimento, 19(1):154-164, 1961.

J. P. Goodridge, P. A. Dudchenko, K. A. Worboys, E. J. Golob, and J. S. Taube. Cue control and head direction cells. Behav. Neurosci., 112(4):749-761, 1998.

J. Green, A. Adachi, K. K. Shah, J. D. Hirokawa, P. S. Magani, and G. Maimon. A neural circuit architecture for angular integration in Drosophila. Nature, 546(7656):101-106, 2017.

Y. Gu, S. Lewallen, A. A. Kinkhabwala, C. Domnisoru, K. Yoon, J. L. Gauthier, I. R. Fiete, and D. W. Tank. A map-like micro-organization of grid cells in the medial entorhinal cortex. Cell, 175(3):736-750, 2018.

A. Guanella, D. Kiper, and P. Verschure. A model of grid cells based on a twisted torus topology. Int. J. Neural Syst., 17(04):231-240, 2007. 
T. Hafting, M. Fyhn, S. Molden, M.-B. Moser, and E. I. Moser. Microstructure of a spatial map in the entorhinal cortex. Nature, 436(7052):801-806, 2005.

K. Hardcastle, S. Ganguli, and L. M. Giocomo. Environmental boundaries as an error correction mechanism for grid cells. Neuron, 86(3):827-839, 2015.

J. J. Hopfield. Neurons with graded response have collective computational properties like those of two-state neurons. Proc. Natl. Acad. Sci. U.S.A., 81(10):3088-3092, 1984.

V. Itskov, D. Hansel, and M. Tsodyks. Short-term facilitation may stabilize parametric working memory trace. Front. Comput. Neurosci., 5:40, 2011.

L. Kang and V. Balasubramanian. A geometric attractor mechanism for self-organization of entorhinal grid modules. eLife, 8:e46687, 2019.

L. Kang and M. R. DeWeese. Replay as wavefronts and theta sequences as bump oscillations in a grid cell attractor network. eLife, 8:e46351, 2019.

M. Khona, S. Chandra, and I. R. Fiete. From smooth cortical gradients to discrete modules: spontaneous and topologically robust emergence of modularity in grid cells. bioRxiv 2021.10.28.466284, 2022.

Z. P. Kilpatrick and B. Ermentrout. Wandering bumps in stochastic neural fields. SIAM J. Appl. Dyn. Syst., 12(1):61-94, 2013.

S. S. Kim, H. Rouault, S. Druckmann, and V. Jayaraman. Ring attractor dynamics in the Drosophila central brain. Science, 356(6340):849-853, 2017.

J. Krupic, M. Bauza, S. Burton, C. Lever, and J. O'Keefe. How environment geometry affects grid cell symmetry and what we can learn from it. Philos. Trans. R. Soc. B, 369(1635):20130188, 2014.

C. K. Machens, R. Romo, and C. D. Brody. Flexible control of mutual inhibition: a neural model of two-interval discrimination. Science, 307(5712):1121-1124, 2005.

S. N. Majumdar, A. Pal, and G. Schehr. Extreme value statistics of correlated random variables: A pedagogical review. Phys. Rep., 840:1-32, 2020.

A. Mathis, A. V. M. Herz, and M. Stemmler. Optimal population codes for space: grid cells outperform place cells. Neural Comput., 24(9):2280-2317, 2012.

B. L. McNaughton, L. L. Chen, and E. J. Markus. Dead reckoning, landmark learning, and the sense of direction: a neurophysiological and computational hypothesis. J. Cognit. Neurosci., 3(2):190-202, 1991.

B. L. McNaughton, F. P. Battaglia, O. Jensen, E. I. Moser, and M.-B. Moser. Path integration and the neural basis of the 'cognitive map'. Nat. Rev. Neurosci., 7(8):663-678, 2006.

J. Milnor. On the concept of attractor. Commun. Math. Phys., 99(2):177-195, 1985.

N. Mosheiff and Y. Burak. Velocity coupling of grid cell modules enables stable embedding of a low dimensional variable in a high dimensional neural attractor. eLife, 8:e48494, 2019.

Y. Nambu. Quasi-particles and gauge invariance in the theory of superconductivity. Phys. Rev., 117(3): 648-663, 1960.

Z. Navratilova, L. M. Giocomo, J.-M. Fellous, M. E. Hasselmo, and B. L. McNaughton. Phase precession and variable spatial scaling in a periodic attractor map model of medial entorhinal grid cells with realistic after-spike dynamics. Hippocampus, 22(4):772-789, 2012.

J. O'Keefe and N. Burgess. Dual phase and rate coding in hippocampal place cells: theoretical significance and relationship to entorhinal grid cells. Hippocampus, 15(7):853-866, 2005. 
A. Renart, P. Song, and X.-J. Wang. Robust spatial working memory through homeostatic synaptic scaling in heterogeneous cortical networks. Neuron, 38(3):473-485, 2003.

A. Samsonovich and B. L. McNaughton. Path integration and cognitive mapping in a continuous attractor neural network model. J. Neurosci., 17(15):5900-5920, 1997.

A. Seeholzer, M. Deger, and W. Gerstner. Stability of working memory in continuous attractor networks under the control of short-term plasticity. PLOS Comput. Biol., 15(4):e1006928, 2019.

J. D. Seelig and V. Jayaraman. Neural dynamics for landmark orientation and angular path integration. Nature, 521(7551):186-191, 2015.

H. S. Seung. How the brain keeps the eyes still. Proc. Natl. Acad. Sci. U.S.A., 93(23):13339-13344, 1996.

W. E. Skaggs, J. J. Knierim, H. S. Kudrimoti, and B. L. McNaughton. A model of the neural basis of the rat's sense of direction. Adv. Neural Inf. Process. Syst., 7:173-180, 1995.

D. C. Somers, S. B. Nelson, and M. Sur. An emergent model of orientation selectivity in cat visual cortical simple cells. J. Neurosci., 15(8):5448-5465, 1995.

B. Sorscher, G. Mel, S. Ganguli, and S. Ocko. A unified theory for the origin of grid cells through the lens of pattern formation. Adv. Neural Inf. Process. Syst., 32:10003-10013, 2019.

S. Sreenivasan and I. Fiete. Grid cells generate an analog error-correcting code for singularly precise neural computation. Nat. Neurosci., 14(10):1330-1337, 2011.

M. Stemmler, A. Mathis, and A. V. M. Herz. Connecting multiple spatial scales to decode the population activity of grid cells. Sci. Adv., 1(11):e1500816-e1500816, 2015.

H. Stensola, T. Stensola, T. Solstad, K. Frøland, M.-B. Moser, and E. I. Moser. The entorhinal grid map is discretized. Nature, 492(7427):72-78, 2012.

S. M. Stringer, T. P. Trappenberg, E. T. Rolls, and I. E. T. d. Araujo. Self-organizing continuous attractor networks and path integration: one-dimensional models of head direction cells. Netw. Comput. Neural Syst., 13(2):217-242, 2002.

S. M. Stringer, E. T. Rolls, and T. P. Trappenberg. Self-organising continuous attractor networks with multiple activity packets, and the representation of space. Neural Networks, 17(1):5-27, 2004.

S. M. Stringer, E. T. Rolls, and T. P. Trappenberg. Self-organizing continuous attractor network models of hippocampal spatial view cells. Neurobiol. Learn. Mem., 83(1):79-92, 2005.

J. S. Taube. The head direction signal: origins and sensory-motor integration. Annu. Rev. Neurosci., 30(1): 181-207, 2007.

J. S. Taube, R. U. Muller, and J. B. Ranck. Head-direction cells recorded from the postsubiculum in freely moving rats. I. Description and quantitative analysis. J. Neurosci., 10(2):420-435, 1990.

K. Thurley, C. Leibold, A. Gundlfinger, D. Schmitz, and R. Kempter. Phase precession through synaptic facilitation. Neural Comput., 20(5):1285-1324, 2008.

M. Tsodyks and T. Sejnowski. Associative memory and hippocampal place cells. Int. J. Neural Syst., 6: S81-S86, 1995.

D. Turner-Evans, S. Wegener, H. Rouault, R. Franconville, T. Wolff, J. D. Seelig, S. Druckmann, and V. Jayaraman. Angular velocity integration in a fly heading circuit. eLife, 6:e04577, 2017.

X.-X. Wei, J. Prentice, and V. Balasubramanian. A principle of economy predicts the functional architecture of grid cells. eLife, 4:e08362, 2015. 
J. Widloski. Grid cell attractor networks: development and implications. Doctoral dissertation, University $73:$ of Texas at Austin, 2015.

J. Widloski, M. P. Marder, and I. R. Fiete. Inferring circuit mechanisms from sparse neural recording and ${ }_{741}$ global perturbation in grid cells. eLife, 7:e33503, 2018.

K. Wimmer, D. Q. Nykamp, C. Constantinidis, and A. Compte. Bump attractor dynamics in prefrontal 743 cortex explains behavioral precision in spatial working memory. Nat. Neurosci., 17(3):431-439, 2014.

S. Wu, S.-i. Amari, and H. Nakahara. Population coding and decoding in a neural field: a computational 745 study. Neural Comput., 14(5):999-1026, 2002.

S. Wu, K. Hamaguchi, and S.-i. Amari. Dynamics and computation of continuous attractors. Neural Comput., ${ }_{747}$ 20(4):994-1025, 2008.

X. Xie, R. H. R. Hahnloser, and H. S. Seung. Double-ring network model of the head-direction system. Phys. $\quad 749$ Rev. E, 66(4):041902, 2002.

K. Yoon, M. A. Buice, C. Barry, R. Hayman, N. Burgess, and I. R. Fiete. Specific evidence of low-dimensional 751 continuous attractor dynamics in grid cells. Nat. Neurosci., 16(8):1077-1084, 2013.

K. Zhang. Representation of spatial orientation by the intrinsic dynamics of the head-direction cell ensemble: ${ }_{753}$ A theory. J. Neurosci., 16(6):2112-2126, 1996. 